\title{
Homogenization of the nonlinear Maxwell model of viscoelasticity and of the Prandtl-Reuss model of elastoplasticity
}

\author{
Augusto Visintin \\ Dipartimento di Matematica, Università degli Studi di Trento, \\ via Sommarive 14, 38050 Povo (Trento), Italy \\ (visintin@science.unitn.it)
}

(MS received 2 June 2006; accepted 24 October 2007)

This paper deals with processes in nonlinear inelastic materials whose constitutive behaviour is represented by the inclusion

$$
\frac{\partial}{\partial t}[\varepsilon-B(x): \sigma] \in \partial \varphi(\sigma, x)
$$

here we denote by $\sigma$ the stress tensor, by $\varepsilon$ the linearized strain tensor, by $B(x)$ the compliance tensor and by $\partial \varphi(\cdot, x)$ the subdifferential of a convex function $\varphi(\cdot, x)$. This relation accounts for elasto-viscoplasticity, including a nonlinear version of the classical Maxwell model of viscoelasticity and the Prandtl-Reuss model of elastoplasticity.

The constitutive law is coupled with the equation of continuum dynamics, and well-posedness is proved for an initial- and boundary-value problem. The function $\varphi$ and the tensor $B$ are then assumed to oscillate periodically with respect to $x$ and, as this period vanishes, a two-scale model of the asymptotic behaviour is derived via Nguetseng's notion of two-scale convergence. A fully homogenized single-scale model is also retrieved, and its equivalence with the two-scale problem is proved. This formulation is non-local in time and is at variance with that based on so-called analogical models that rest on a mean-field-type hypothesis.

\section{Introduction}

In this paper we deal with processes in multi-axial spatially distributed inelastic media. First, we prove the well-posedness of an initial- and boundary-value problem that accounts for nonlinear viscoelasticity, including the Prandtl-Reuss model of elastoplasticity as a limit case. We then assume that the material is mesoscopically inhomogeneous and derive a homogenized (i.e. effective) model. The conclusions that we attain are at variance with a largely used approach based on analogical models.

\subsection{The basic model}

We denote by $\boldsymbol{u}$ the displacement with respect to the initial configuration $\Omega$ $\left(\Omega \subset \mathbb{R}^{3}\right)$, by $\varepsilon$ the linearized strain tensor, by $\sigma$ the stress tensor, by $\rho$ the density and by $f$ a spatially distributed load. We also couple the equation of continuum

(c) 2008 The Royal Society of Edinburgh 
dynamics

$$
\left.\rho \frac{\partial^{2} \boldsymbol{u}}{\partial t^{2}}-\nabla \cdot \sigma=\boldsymbol{f} \quad \text { in } \Omega \times\right] 0, T[
$$

with initial and boundary conditions. We then formulate the constitutive relation, assuming the hypothesis of infinitesimal displacements and using the language of convex analysis (see, for example, $[39,50,52,72,84]$ ). More specifically, we fix a fourth-order compliance tensor $B(x)$ and a lower semicontinuous convex function $\varphi(\cdot, x)$, denote its subdifferential by $\partial \varphi(\cdot, x)$ and consider the inclusion

$$
\frac{\partial \varepsilon}{\partial t}-B(x): \frac{\partial \sigma}{\partial t} \in \partial \varphi(\sigma, x)
$$

This relation may represent a large class of elasto-viscoplastic behaviours. For a quadratic function $\varphi(\cdot, x)$, the inclusion (1.2) is reduced to the classical (linear) Maxwell model of viscoelasticity of fluid type (see, for example, [42, 47, 62, 63, 82]). If the support of $\varphi$ is bounded, one retrieves the Prandtl-Reuss model of elastoplasticity without strain-hardening. In this case (1.2) also accounts for the possible occurrence of moving interfaces, i.e. free boundaries, that separate the elastic and plastic phases, where the strain may concentrate. It is known that in this latter case the (non-reflexive) Banach space of Radon measures is the most natural functional framework, rather than any Sobolev space.

In dealing with vibration phenomena and with other rapid processes, it is appropriate to use equation (1.1), whereas in several other cases the time-scale of this equation is much shorter than that of the constitutive law (1.2). By passing to the limit as $\rho \rightarrow 0$ in the system (1.1), (1.2), we then retrieve the problem in which (1.1) is replaced by the quasi-static equation $-\nabla \cdot \sigma=\boldsymbol{f}$.

\subsection{Generalized (nonlinear) Maxwell model}

Rheological models are often represented via finite networks of springs, dashpots and other either linear or nonlinear elements. In this framework the Maxwell model may be represented as the series arrangement of an elastic component and a viscous one. After [63] the parallel combination of a family of Maxwell models is labelled as a generalized Maxwell model; the nonlinearity of the viscous element does not modify this construction, which we now outline.

Let us assume that the tensor $B$ and the function $\varphi$ depend on an index $j \in$ $\{1, \ldots, M\}$, and let us represent the solution of the Cauchy problem associated to $(1.2)$ in the form $\sigma_{j}=\mathcal{G}_{j}\left(\varepsilon_{j}\right), \mathcal{G}_{j}$ being a hereditary operator (that also depends on the initial value $\sigma(0)$ which is omitted here). We thus have a family of $M$ elementary models, each one characterized by an operator $\mathcal{G}_{j}$; somehow schematically, each of them may be thought of as representing a grain of a composite material. According to the assembling rules of analogical models, if the system is univariate and these elements are arranged in parallel, then their strains are uniform and coincide with the strain $\varepsilon$ of the composed model, whereas the stress of the latter equals the sum of the stresses $\sigma_{j}$, that is,

$$
\varepsilon=\varepsilon_{j} \quad \text { for all } j, \quad \sigma=\sum_{j=1}^{M} \sigma_{j}=\sum_{j=1}^{M} \mathcal{G}_{j}(\varepsilon)=: \tilde{\mathcal{G}}(\varepsilon)
$$


Because of the hypothesis of uniformity of the strain, this may be regarded as a mean-field model.

This formulation may be extended to the multivariate setting, although in this case the interpretation in terms of parallel arrangement fails. This construction is also easily extended if a continuous distribution of elements is set at an underlying finer length-scale, which we represent by means of a variable $y$ ranging through a reference volume element $Y:=\left[0,1\left[^{3}\right.\right.$. In this case it suffices to replace (1.3) by the conditions

$$
\varepsilon \text { is uniform in } Y, \quad \sigma=\int_{Y} \sigma(y) \mu(y) \mathrm{d} y=\int_{Y} \mathcal{G}(\varepsilon, y) \mu(y) \mathrm{d} y=: \hat{\mathcal{G}}(\varepsilon),
$$

where $\mu$ is a prescribed weight function.

\subsection{Two-scale and single-scale homogenization}

The significance of the above analogical model seems to be essentially heuristic. However, as it is based on the composition of a family of elements, we wonder whether it may be retrieved from some underlying fine-scale structure via a homogenization procedure. We thus deal with the system (1.1), (1.2) assuming that the tensor $B$ and the function $\varphi$ are (possibly discontinuous) periodic functions of $x / \eta$, with $0<\eta \ll 1$; for instance, a composite material may correspond to a piecewiseconstant dependence on $x / \eta$. We then let the parameter $\eta$ vanish and derive a two-length-scale model via Nguetseng's notion of two-scale convergence [80]. We complete the homogenization procedure by proving the equivalence between the latter two-scale model and a single-scale problem: this is the main result of this paper. The outcome of this analysis is at variance with the mean-field hypothesis that underlies the generalized Maxwell model (see (1.4)). The single-scale constitutive relation that we derive is non-local in time, and it is not clear whether it might be rewritten as a gradient flow like (6.25). This is reminiscent of memory effects that are known to occur in the homogenization of linear evolution equations (see $[95,96])$.

Let us now outline the structure of the paper. In $\S 2$ we illustrate the constitutive relation (1.2) for a macroscopically non-homogeneous material. In $\S 3$ we formulate an initial- and boundary-value problem for the system (1.1), (1.2) in the framework of Sobolev spaces. In $\S 4$ we then prove the well-posedness of this problem and illustrate how the existence proof may be amended for the limit case of the PrandtlReuss model. In particular, this requires the introduction of a space analogous to that of functions with bounded deformation, $\mathrm{BD}(\Omega)$. In $\S 5$ we derive a twoscale model via two-scale convergence; here some modifications are also needed for the Prandtl-Reuss material. In $\S 6$ we homogenize the constitutive relation (1.2) under the restriction that $\varphi$ grows quadratically. In $\S 7$ we retrieve a purely coarsescale problem under the same restriction and show its equivalence to the two-scale formulation. Finally, in $\S 8$ we draw our conclusions and point out some further questions.

\subsection{Literature}

Many works deal with the analysis of viscoelasticity and elastoplasticity; here is just a partial list: $[1-3,9,36,42,46-49,57,58,62-64,67-69,72-74,78,79,82,83,86,92$, 
97]. In particular, the Prandtl-Reuss model has been dealt with in, for example, $[10,11,26,36,40,48,54-56,79,90,92,98]$. Our proof of existence of a weak solution rests on classical techniques (see, for example, $[36,48,79])$ and in the limit case of elastoplastic materials also uses the Banach space $\operatorname{BD}(\Omega)$ of functions with bounded deformation (see, for example, [7, 88, 89, 91, 97, 98]). A thorough formulation of the Prandtl-Reuss model in $\operatorname{BD}(\Omega)$ has recently been provided in [32]. In [13, 14] existence and uniqueness of the solution in the framework of Hilbert spaces were proved for a more general constitutive law than (1.2) (see also [61]). The construction via analogical models is the basis of the Prandtl-Ishlinskiu models of stop-type, which extend the Prandtl-Reuss model and have been studied in the theory of hysteresis (see, for example, $[24,59,60,100]$ ).

As we have already pointed out, convex analysis plays a key role in this paper. In particular, the Fenchel properties (2.12) and (2.13) are at the basis of our weak formulation, which may be compared with that of $[22,23]$. This approach is also used here for homogenization.

A large number of works devoted to homogenization have been published since the seminal articles $[34,87,94]$ that were at the origin of the De Giorgi theory of $\Gamma$-convergence $[20,21,31,33]$ and of the Tartar-Murat theory of compensated compactness $[75,76,94]$. Homogenization was applied to continuum mechanics in, for example, $[5,12,27,30,51,53,71,76,85]$. The approach based on the notion of two-scale convergence was first pioneered by Nguetseng [80] and then developed by Allaire [4]; afterwards it was applied in many works (see, for example, [65] for a recent review).

The homogenization of plasticity has been studied in, for example, $[15-19,35,38$, $92,93]$. The method of two-scale convergence has recently been applied to this phenomenon. In [28] it was used for the (stationary) Hencky model of elastoplasticity, namely for a stationary variational inequality. Quasi-stationary processes for a wide class of inelastic materials were dealt with in $[3,78]$; this class includes viscoplasticity and the Prandtl-Reuss model, as well as other constitutive laws of engineering interest that are not representable via variational inequalities. The latter two papers dealt with homogenization via two-scale homogenization, via an approach that is at variance with that of the present work. A different formulation of quasi-static elastoplastic processes with strain-hardening and their two-scale homogenization were treated [70], via the energetic approach to rate-independent evolution. The procedure of the present work for the homogenization of the Prandtl-Reuss model rests on the extension of the notion of two-scale convergence to measures, that was introduced and studied by Amar in [6]. Different extensions can be found, for example, in $[37,99,108]$.

In the duality between parallel and series arrangements, the dual model of the inclusion (1.2) reads

$$
\frac{\partial \varepsilon}{\partial t} \in \partial \varphi\left(\sigma-B(y)^{-1}: \varepsilon\right) .
$$

This is a nonlinear version of the classical Kelvin-Voigt model of viscoelasticity of solid type; for a suitable selection of the function $\varphi,(1.5)$ also accounts for the Prager model of rigid plasticity with linear kinematic strain-hardening (see, for example, $[42,47,63,82])$. The approach of the present work, based on two- and single-scale formulations of the homogenized problem, was applied to the latter 
model in [103]. In [43] the homogenization of the Prager model was also studied for univariate systems via hysteresis operators (in passing we point out that this analysis might easily be extended to the nonlinear Kelvin-Voigt model).

The present work is part of research on the two-scale homogenization of nonlinear models of continuum mechanics, electromagnetism and heat conduction (see [102, $103,106,107])$.

\section{Constitutive law}

In this section we outline the rheological models that we deal with in this paper.

We denote by $\mathbb{R}^{9}$ the linear space of the $3 \times 3$-tensors and by $\mathbb{R}_{\mathrm{s}}^{9}$ the linear subspace of symmetric tensors. We mark the vectors of $\mathbb{R}^{3}$ in bold, but use no special symbol for higher-order tensors; we denote the scalar product by '.' and the contraction over two indices by ':' (i.e. $u: v:=\sum_{i, j=1}^{3} u_{i j} v_{i j}$ for any $u, v \in \mathbb{R}^{9}$ ). For any tensor $v \in \mathbb{R}^{9}$ we label its spheric and deviatoric components by the indices '( $\mathrm{s}$ )' and '(d)', respectively. We write $L^{2}(\Omega)_{\mathrm{s}}^{9}$ in place of $L^{2}\left(\Omega ; \mathbb{R}_{\mathrm{s}}^{9}\right)$, and similar expressions for other function spaces; we also denote by $D_{\mathrm{s}}^{9}$ the linear space of symmetric $3 \times 3$ deviatoric tensors.

We denote the displacement field by $\boldsymbol{u}$ and the stress tensor by $\sigma$. Under the assumption of infinitesimal displacements we define the linearized strain tensor by

$$
\varepsilon_{i j}:=\left(\nabla^{\mathrm{s}} \boldsymbol{u}\right)_{i j}=\frac{1}{2}\left(\frac{\partial u_{i}}{\partial x_{j}}+\frac{\partial u_{j}}{\partial x_{i}}\right) \text { for } i, j=1,2,3 .
$$

We assume a linear elastic relation between the spheric components of the strain and stress tensors

$$
\varepsilon_{(\mathrm{s})}=\beta \sigma_{(\mathrm{s})} \quad \text { for a constant } \beta>0,
$$

and for the deviatoric components we first consider the relation

$$
\frac{\partial \varepsilon_{(\mathrm{d})}}{\partial t} \in \partial \tilde{\varphi}\left(\sigma_{(\mathrm{d})}\right)\left(\subset D_{\mathrm{s}}^{9}\right),
$$

where $\tilde{\varphi}: \mathbb{R}_{\mathrm{s}}^{9} \rightarrow \mathbb{R} \cup\{+\infty\}$ is a prescribed convex function. This may account for a number of basic constitutive behaviours, e.g.

(i) linear shear viscosity, if $\tilde{\varphi}$ is quadratic;

(ii) nonlinear shear viscosity: this encompasses the Norton and Bingham materials, which may respectively be represented by

$$
\left.\begin{array}{ll}
\tilde{\varphi}_{N}(v):=c|v|^{a} & \text { for some } c>0 \text { and } a>1, \\
\tilde{\varphi}_{B}(v):=d|v|^{2}+e|v| & \text { for some } d, e>0,
\end{array}\right\}
$$

for any $v \in \mathbb{R}_{\mathrm{s}}^{9}$ (see, for example, $[2,36,44,63,64,97]$ );

(iii) rigid plasticity without strain-hardening, if $\tilde{\varphi}=I_{K}$ is the indicator function of a closed convex set $K \subset D_{\mathrm{s}}^{9}$ that includes the origin: namely $I_{K}(v):=0$ if $v \in K$ and $I_{K}(v):=+\infty$ otherwise (for instance, $K$ might be either von Mises's or Tresca's yield criteria); 
(iv) viscoplasticity, 'in its classical meaning (see [81]) [namely] rate-dependent behaviour with a well-defined yield criterion' [64, p. 102], if $\varphi$ is linear outside a closed convex set $K \subset D_{\mathrm{s}}^{9}$ as above.

By the definition of the subdifferential, the inclusion (2.3) is equivalent to the variational inequality

$$
\frac{\partial \varepsilon_{(\mathrm{d})}}{\partial t} \cdot\left(\sigma_{(\mathrm{d})}-v\right) \geqslant \tilde{\varphi}\left(\sigma_{(\mathrm{d})}\right)-\tilde{\varphi}(v) \quad \text { for all } v \in \mathbb{R}^{9} ;
$$

for $\tilde{\varphi}=I_{K}$ this also reads

$$
\sigma \in K, \quad \frac{\partial \varepsilon_{(\mathrm{d})}}{\partial t} \cdot\left(\sigma_{(\mathrm{d})}-v\right) \geqslant 0 \quad \text { for all } v \in K .
$$

Let us now fix a constant, fourth-order compliance tensor $B$, and assume that, denoting by the Kronecker symbol $\delta_{i j}$ the components of the $3 \times 3$-identity tensor,

$$
\left.\begin{array}{rlrl}
B_{i j k \ell} & =B_{j i k \ell}=B_{k \ell i j} & & \text { for all } i, j, k, \ell \in\{1,2,3\}, \\
\sum_{k=1,2,3} B_{i j k k} & =\beta \delta_{i j} & & \text { for all } i, j \in\{1,2,3\}, \text { for some } \beta>0 .
\end{array}\right\}
$$

Hence, $\sum_{i=1,2,3} B_{i i k \ell}=\beta \delta_{k \ell}$ for any $k$, $\ell$. The transformation $v \mapsto B: v$ then maps spheric (deviatoric, respectively) tensors to spheric (deviatoric, respectively) tensors. Let us now assume that

$$
\left.\begin{array}{c}
\varphi: \mathbb{R}_{\mathrm{s}}^{9} \rightarrow \mathbb{R} \cup\{+\infty\} \text { is lower semicontinuous and convex, } \\
\varphi(v)=\varphi\left(v_{(\mathrm{d})}\right) \quad \text { for all } v \in \mathbb{R}_{\mathrm{s}}^{9},
\end{array}\right\}
$$

and deal with the inclusion

$$
\frac{\partial \varepsilon}{\partial t}-B: \frac{\partial \sigma}{\partial t} \in \partial \varphi(\sigma) \quad\left(\text { here }\left(B: \frac{\partial \sigma}{\partial t}\right)_{i j}:=\sum_{k, \ell=1,2,3} B_{i j k \ell} \frac{\partial \sigma_{k \ell}}{\partial t}\right) .
$$

This accounts for a nonlinear extension of the classical Maxwell model of viscoelasticity, and also for the Prandtl-Reuss model of elastoplasticity if $\varphi=I_{K}$, for a convex set $K \subset D_{\mathrm{s}}^{9}$ as above.

By (2.6) and $(2.7)_{2}$ the inclusion (2.8) is equivalent to the system

$$
\begin{gathered}
\frac{\partial \varepsilon_{(\mathrm{s})}}{\partial t}-\beta \frac{\partial \sigma_{(\mathrm{s})}}{\partial t}=0 \\
\frac{\partial \varepsilon_{(\mathrm{d})}}{\partial t}-B: \frac{\partial \sigma_{(\mathrm{d})}}{\partial t} \in \partial \varphi\left(\sigma_{(\mathrm{d})}\right) .
\end{gathered}
$$

Note that (2.9) is equivalent to (2.2), provided that (2.2) itself is satisfied for $t=0$.

We shall denote by $\mathcal{M}$ the linear subspace of $\mathbb{R}^{3^{4}}$ spanned by the fourth-order tensors that satisfy (2.6), and assume that $B \in \mathcal{M}$ and is positive definite.

\subsection{Fenchel's properties}

In view of interpreting (2.8) we briefly review a classical construction of convex analysis (see, for example, $[39,50,52,72,84]$ ). For any $N \in \mathbb{N}$, let $F: \mathbb{R}^{N} \rightarrow \mathbb{R} \cup\{+\infty\}$ 
be any function such that $F \not \equiv+\infty$; identify $\mathbb{R}^{N}$ with its dual space and define the convex conjugate function

$$
F^{*}: \mathbb{R}^{N} \rightarrow \mathbb{R} \cup\{+\infty\}: w \mapsto \sup \left\{w \cdot v-F(v): v \in \mathbb{R}^{N}\right\} .
$$

For instance, for $F=I_{K}$ with $K$ as above, $I_{K}^{*}(w)=\sup \{w \cdot v: v \in K\}$ for any $w \in \mathbb{R}^{N} ; I_{K}^{*}$ is convex and positively homogeneous of degree 1 , and is named the support function of the set $K$. After [41], for any $u, w \in \mathbb{R}^{N}$,

$$
\begin{aligned}
F(u)+F^{*}(w) & \geqslant w \cdot u & & \text { (Fenchel inequality) }, \\
w \in \partial F(u) & \Longleftrightarrow F(u)+F^{*}(w)=w \cdot u & & \text { (Fenchel property I). }
\end{aligned}
$$

Because of (2.12) the latter statement also reads

$$
w \in \partial F(u) \Longleftrightarrow F(u)+F^{*}(w) \leqslant w \cdot u \quad \text { (Fenchel property II). }
$$

This setting found an interesting application in $[22,23]$ in connection with evolution.

\subsection{The power balance}

By (2.13) the inclusion (2.8) is equivalent to

$$
\varphi(\sigma)+\varphi^{*}\left(\frac{\partial \varepsilon}{\partial t}-B: \frac{\partial \sigma}{\partial t}\right)=\sigma:\left(\frac{\partial \varepsilon}{\partial t}-B: \frac{\partial \sigma}{\partial t}\right)
$$

that is,

$$
\sigma: \frac{\partial \varepsilon}{\partial t}=\varphi(\sigma)+\varphi^{*}\left(\frac{\partial \varepsilon}{\partial t}-B: \frac{\partial \sigma}{\partial t}\right)+\frac{1}{2} \frac{\partial}{\partial t}(\sigma: B: \sigma) .
$$

This equality accounts for the pointwise power balance: the power provided by the stress, $\sigma: \partial \varepsilon / \partial t$, equals the sum of the power dissipated by viscosity, $\varphi(\sigma)+$ $\varphi^{*}(\partial \varepsilon / \partial t-B: \partial \sigma / \partial t)$ and the elastic power, $\frac{1}{2} \partial(\sigma: B: \sigma) / \partial t$. Although physically the dissipation cannot be negative, for our analysis we shall not need this hypothesis: the inferior boundedness will suffice. Because of (2.14) the equality (2.15) is also equivalent to the inequality

$$
\sigma: \frac{\partial \varepsilon}{\partial t} \geqslant \varphi(\sigma)+\varphi^{*}\left(\frac{\partial \varepsilon}{\partial t}-B: \frac{\partial \sigma}{\partial t}\right)+\frac{1}{2} \frac{\partial}{\partial t}(\sigma: B: \sigma) .
$$

The energy balance may thus be represented in the form either of an inequality or of an equality. In this paper we shall encounter several examples of equivalence between an inequality and the corresponding equality: all of them will stem from (2.13) and (2.14). Finally, note that (2.16) may also be restated as what could be named a null minimization problem:

$$
\begin{aligned}
& J(\sigma, \varepsilon)=\inf J=0, \quad \text { where } \\
& J(\sigma, \varepsilon):=\iint_{\Omega_{T}}\left\{\varphi(\sigma)+\varphi^{*}\left(\frac{\partial \varepsilon}{\partial t}-B: \frac{\partial \sigma}{\partial t}\right)+\frac{1}{2} \frac{\partial}{\partial t}(\sigma: B: \sigma)-\sigma: \frac{\partial \varepsilon}{\partial t}\right\} \mathrm{d} x \mathrm{~d} t .
\end{aligned}
$$

In $[106,107]$ a remark like this is used to reformulate other nonlinear models of continuum mechanics as minimization problems. 


\section{Weak formulation}

In this section we provide the weak formulation of an initial- and boundary-value problem that accounts for processes in an inhomogeneous nonlinear viscoelastic material represented by the constitutive inclusion (2.8). This also encompasses the Prandtl-Reuss model of elastoplasticity as a particular case, which requires a substantial modification of the functional framework that we shall illustrate afterwards.

We consider a material of density $\rho=\rho(x)$ that occupies a bounded domain $\Omega \subset \mathbb{R}^{3}$, fix any $T>0$, set $\left.A_{t}:=\Omega \times\right] 0, t[$ for any set $A$ and any $\left.t \in] 0, T\right]$. We fix a partition $\left\{\Gamma_{0}, \Gamma_{1}\right\}$ of the boundary of $\Omega$; we assume that a load $\boldsymbol{f}_{1}$ and a traction $\boldsymbol{g}$ are respectively applied to the bulk of $\Omega$ and to $\Gamma_{1}$, whereas $\Gamma_{0}$ is kept fixed, and that

$$
\begin{gathered}
\rho \in L^{\infty}(\Omega), \quad \rho \geqslant \text { const. }>0 \text { almost everywhere (a.e.) in } \Omega, \\
B \in L^{\infty}(\Omega ; \mathcal{M}), \quad \exists \bar{c}>0: \forall v \in \mathbb{R}_{\mathrm{s}}^{9}, v: B(x): v \geqslant \bar{c}|v|^{2} \text { for a.e. } x \in \Omega, \\
\varphi: \mathbb{R}_{\mathrm{s}}^{9} \times \Omega \rightarrow \mathbb{R} \cup\{+\infty\}, \\
v \mapsto \varphi(v, x) \text { is convex and lower semicontinuous for a.e. } x, \\
x \mapsto \varphi(v, x) \text { is measurable for any } v \\
\left\{v \in \mathbb{R}_{\mathrm{s}}^{9}: \varphi(v, x)<+\infty\right\} \text { has non-empty interior for a.e. } x \\
\varphi(v, x)=\varphi\left(v_{(\mathrm{d})}, x\right) \text { for all } v \in \mathbb{R}_{\mathrm{s}}^{9} \text {, for a.e. } x \text {. }
\end{gathered}
$$

(We shall often omit the $\operatorname{arguments} x$ and $t$ in formulae.) We couple the constitutive law

$$
\frac{\partial \varepsilon}{\partial t}-B(x): \frac{\partial \sigma}{\partial t} \in \partial \varphi(\sigma, x) \text { in } \Omega_{T}
$$

with the equation of continuum dynamics

$$
\rho \frac{\partial^{2} \boldsymbol{u}}{\partial t^{2}}-\nabla \cdot \sigma=\boldsymbol{f}_{1} \quad \text { in } \Omega_{T} \quad\left(\text { here }(\nabla \cdot \sigma)_{i}:=\sum_{j=1}^{3} \frac{\partial \sigma_{i j}}{\partial x_{j}} \text { for } i=1,2,3\right)
$$

we denote by $\boldsymbol{\nu}$ the outward-oriented unit normal vector, and prescribe the initial and boundary conditions

$$
\begin{gathered}
\boldsymbol{u}(\cdot, 0)=\boldsymbol{u}^{0}, \quad \frac{\partial \boldsymbol{u}}{\partial t}(\cdot, 0)=\boldsymbol{v}^{0} \quad \text { in } \Omega, \\
\boldsymbol{u}=\mathbf{0} \quad \text { on } \Gamma_{0 T}, \\
\sigma \cdot \boldsymbol{\nu}=\boldsymbol{g} \quad \text { on } \Gamma_{1 T} \quad\left(\text { i.e. } \sum_{j=1}^{3} \sigma_{i j} \nu_{j}=g_{i} \text { for } i=1,2,3\right),
\end{gathered}
$$

for given fields $\boldsymbol{u}^{0}, \boldsymbol{v}^{0}, \boldsymbol{g}$. Multiplying (3.6) scalarly by $\boldsymbol{v}:=\partial \boldsymbol{u} / \partial t$ and integrating over $\Omega$, we get

$$
\left.\frac{1}{2} \frac{\mathrm{d}}{\mathrm{d} t} \int_{\Omega} \rho|\boldsymbol{v}|^{2} \mathrm{~d} x+\int_{\Omega} \sigma: \frac{\partial \varepsilon}{\partial t} \mathrm{~d} x=\int_{\Omega} \boldsymbol{f}_{1} \cdot \boldsymbol{v} \mathrm{d} x+\int_{\Gamma_{1}} \boldsymbol{g} \cdot \boldsymbol{v} \mathrm{d} S \quad \text { in }\right] 0, T[.
$$


(We denote the elementary surface area by $\mathrm{d} S$.) We write the constitutive law (3.5) in the form of the inequality (2.16), which we saw to represent the pointwise power balance. By (3.10), (2.16) is equivalent to

$$
\begin{aligned}
\frac{1}{2} \frac{\mathrm{d}}{\mathrm{d} t} \int_{\Omega}\left(\rho|\boldsymbol{v}|^{2}+\frac{1}{2} \sigma: B(x): \sigma\right) \mathrm{d} x & +\int_{\Omega}\left[\varphi(\sigma, x)+\varphi^{*}\left(\frac{\partial \varepsilon}{\partial t}-B(x): \frac{\partial \sigma}{\partial t}, x\right)\right] \mathrm{d} x \\
& \left.\leqslant \int_{\Omega} \boldsymbol{f}_{1} \cdot \boldsymbol{v} \mathrm{d} x+\int_{\Gamma_{1}} \boldsymbol{g} \cdot \boldsymbol{v} \mathrm{d} S \text { in }\right] 0, T[.
\end{aligned}
$$

By (2.12) and (2.13) this inequality is in turn equivalent to the corresponding equality that represents the global power balance.

\subsection{Weak formulation}

We assume that the (bounded) domain $\Omega$ is of Lipschitz class and that $\Gamma_{0}$ is measurable and has positive bi-dimensional Hausdorff measure. We fix any $p \in$ $\left[2,+\infty\left[\right.\right.$ and set $q:=p /(p-1)$, so that $p^{-1}+q^{-1}=1$. Omitting the trace operator, we also set

$$
V:=\left\{\boldsymbol{v} \in W^{1, q}(\Omega)^{3}: \boldsymbol{v}=\mathbf{0} \text { on } \Gamma_{0}\right\}, \quad\|\boldsymbol{v}\|_{V}:=\left\|\nabla^{\mathrm{s}} \boldsymbol{v}\right\|_{L^{q}(\Omega)^{9}} .
$$

By the classical Korn and Poincaré inequalities (for the extension of the former to $W^{1, q}(\Omega)^{3}$ see, for example, [44,97]), \|\|$_{V}$ is equivalent to the usual norm of $W^{1, q}(\Omega)^{3}$, so that $V$ is a closed Banach subspace of the Sobolev space $W^{1, q}(\Omega)^{3}$. Identifying $L^{p}(\Omega)^{3}$ with a subspace of $V^{\prime}$ (the dual space of $V$ ), we get

$V \subset L^{q}(\Omega)^{3}, L^{p}(\Omega)^{3} \subset V^{\prime}$ with compact, continuous and dense injections.

We also introduce the space

$V_{2}:=V \cap H^{1}(\Omega)^{3}:$ Hilbert space equipped with the norm $\|\boldsymbol{v}\|_{V_{2}}:=\left\|\nabla^{\mathrm{s}} \boldsymbol{v}\right\|_{L^{2}(\Omega)^{9}}$.

We denote by $\langle\cdot, \cdot\rangle$ the duality pairing between $V^{\prime}$ and $V$, and define the linear and continuous operator

$$
\nabla^{*} \cdot: L^{p}(\Omega)^{9} \rightarrow V^{\prime}, \quad\left\langle\nabla^{*} \cdot w, \boldsymbol{v}\right\rangle:=-\int_{\Omega} w: \nabla^{\mathrm{s}} \boldsymbol{v} \mathrm{d} x \quad \forall w \in L^{p}(\Omega)^{9}, \forall \boldsymbol{v} \in V .
$$

We assume that (3.1)-(3.4) are satisfied and that

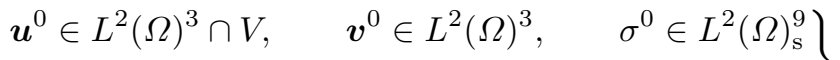

$$
\begin{aligned}
& \text { such that, setting } \left.\varepsilon^{0}:=\nabla^{\mathrm{s}} \boldsymbol{u}^{0}, \quad \varepsilon_{(\mathrm{s})}^{0}=\beta \sigma_{(\mathrm{s})}^{0}, \quad\right\} \\
& \boldsymbol{f}:=\boldsymbol{f}_{1}+\boldsymbol{f}_{2}, \quad \boldsymbol{f}_{1} \in L^{p}\left(0, T ; L^{2}(\Omega)^{3}\right), \quad \boldsymbol{f}_{2} \in W^{1, p}\left(0, T ; V^{\prime}\right), \\
& \left.\begin{array}{c}
\exists a, b, a_{1}, b_{1}>0 \text { : for all } v \in \mathbb{R}_{\mathrm{s}}^{9} \text {, for a.e. } x \in \Omega, \\
a\left|v_{(\mathrm{d})}\right|^{p}-b \leqslant \varphi(v, x) \leqslant a_{1}\left|v_{(\mathrm{d})}\right|^{p}+b_{1} .
\end{array}\right\}
\end{aligned}
$$

so that, recalling $(3.4)$,

$$
\exists c, d>0 \text { : for all } v \in \mathbb{R}_{\mathrm{s}}^{9} \text {, for a.e. } x \in \Omega, \quad \varphi^{*}(v, x) \geqslant c|v|^{q}-d .
$$


Note that (3.4) excludes the possibility that $\varphi(\cdot, x)$ might be coercive with respect to the spheric component of its argument, and implies that $\varphi^{*}(v, x)$ is finite only if $v$ is a deviator.

Now we are able to introduce a weak formulation of the system (3.5)-(3.9).

Problem 3.1. Find $(\boldsymbol{u}, \sigma)$ such that, setting $\varepsilon:=\nabla^{\mathrm{s}} \boldsymbol{u}$,

$$
\begin{gathered}
\boldsymbol{u} \in W^{1, \infty}\left(0, T ; L^{2}(\Omega)^{3}\right) \cap L^{q}(0, T ; V), \quad \sigma \in L^{\infty}\left(0, T ; L^{2}(\Omega)_{\mathrm{s}}^{9}\right), \\
\sigma_{(\mathrm{d})} \in L^{p}\left(\Omega_{T}\right)_{\mathrm{s}}^{9}, \quad \frac{\partial}{\partial t}(\varepsilon-B(x): \sigma) \in L^{q}\left(\Omega_{T}\right)_{\mathrm{s}}^{9}, \\
\frac{1}{2} \int_{\Omega}\left(\rho\left|\frac{\partial \boldsymbol{u}}{\partial t}(x, t)\right|^{2}+\sigma(x, t): B(x): \sigma(x, t)-\rho\left|\boldsymbol{v}^{0}(x)\right|^{2}-\sigma^{0}(x): B(x): \sigma^{0}(x)\right) \mathrm{d} x \\
+\iint_{\Omega_{t}}\left[\varphi(\sigma, x)+\varphi^{*}\left(\frac{\partial}{\partial \tau}(\varepsilon-B(x): \sigma), x\right)\right] \mathrm{d} x \mathrm{~d} \tau \\
\leqslant \iint_{\Omega_{t}} \boldsymbol{f}_{1} \cdot \frac{\partial \boldsymbol{u}}{\partial \tau} \mathrm{d} x \mathrm{~d} \tau+\left\langle\boldsymbol{f}_{2}(t), \boldsymbol{u}(\cdot, t)\right\rangle-\left\langle\boldsymbol{f}_{2}(0), \boldsymbol{u}^{0}\right\rangle \\
\left.\left.-\int_{0}^{t}\left\langle\frac{\partial \boldsymbol{f}_{2}}{\partial \tau}, \boldsymbol{u}\right\rangle \mathrm{d} \tau \quad \text { for a.e. } t \in\right] 0, T\right], \\
\iint_{\Omega_{T}}\left\{\rho\left(\boldsymbol{u}^{0}-\boldsymbol{u}\right) \cdot \frac{\partial \boldsymbol{w}}{\partial t}+\int_{0}^{t} \sigma(\cdot, \tau) \mathrm{d} \tau: \nabla \boldsymbol{w}\right\} \mathrm{d} x \mathrm{~d} t \\
=\int_{0}^{T}\left\langle\int_{0}^{t} \boldsymbol{f}(\cdot, \tau) \mathrm{d} \tau+\rho \boldsymbol{v}^{0}, \boldsymbol{w}\right\rangle \mathrm{d} t \\
\text { for all } \boldsymbol{w} \in H^{1}\left(0, T ; V_{2}\right), \quad \boldsymbol{w}(\cdot, T)=\mathbf{0} \text { a.e. in } \Omega .
\end{gathered}
$$

\subsection{Interpretation}

As $\varphi(\cdot, x)$ and $\varphi^{*}(\cdot, x)$ are bounded from below, the integrability of the functions $\varphi(\sigma, x)$ and $\varphi^{*}(\partial[\varepsilon-B(x): \sigma] / \partial t, x)$ is implicit in (3.18). By (3.14) and (3.15) this also implies the regularity (3.17). Equation (3.19) yields

$$
\left.\rho \frac{\partial \boldsymbol{u}}{\partial t}-\nabla^{*} \cdot \int_{0}^{t} \sigma(\cdot, \tau) \mathrm{d} \tau=\int_{0}^{t} \boldsymbol{f}(\cdot, \tau) \mathrm{d} \tau+\rho \boldsymbol{v}^{0} \quad \text { in } V_{2}^{\prime} \text { a.e. in }\right] 0, T[.
$$

By comparing the terms of this equality we see that $\partial \boldsymbol{u} / \partial t \in W^{1, p}\left(0, T ; V_{2}^{\prime}\right)$; this yields

$$
\left.\rho \frac{\partial^{2} \boldsymbol{u}}{\partial t^{2}}-\nabla^{*} \cdot \sigma=\boldsymbol{f} \quad \text { in } V_{2}^{\prime} \text { a.e. in }\right] 0, T[,
$$

with the initial conditions (3.7). Equation (3.20) is a weak formulation of (3.6) and (3.9), provided that

$$
\left.\boldsymbol{g} \in W^{1, p}\left(0, T ; L^{p}\left(\Gamma_{1}\right)^{3}\right), \quad\left\langle\boldsymbol{f}_{2}, \boldsymbol{w}\right\rangle:=\int_{\Gamma_{1}} \boldsymbol{g} \cdot \boldsymbol{w} \mathrm{d} S \quad \text { for all } \boldsymbol{w} \in V \text { a.e. in }\right] 0, T[.
$$


Let us now assume that $\partial \boldsymbol{u} / \partial t \in L^{q}\left(0, T ; V_{2}\right)$, so that we may multiply (3.20) by $\partial \boldsymbol{u} / \partial t$; this yields

$$
\begin{aligned}
\frac{1}{2} \int_{\Omega} \rho\left(\left|\frac{\partial \boldsymbol{u}}{\partial t}(x, t)\right|^{2}\right. & \left.-\left|\boldsymbol{v}^{0}(x)\right|^{2}\right) \mathrm{d} x+\iint_{\Omega_{t}} \sigma: \frac{\partial \varepsilon}{\partial t} \mathrm{~d} x \mathrm{~d} \tau \\
=\iint_{\Omega_{t}} \boldsymbol{f}_{1} \cdot \frac{\partial \boldsymbol{u}}{\partial \tau} \mathrm{d} x \mathrm{~d} \tau+\left\langle\boldsymbol{f}_{2}(t), \boldsymbol{u}(\cdot, t)\right\rangle-\left\langle\boldsymbol{f}_{2}(0), \boldsymbol{u}^{0}\right\rangle & \\
& \left.\left.-\int_{0}^{t}\left\langle\frac{\partial \boldsymbol{f}_{2}}{\partial \tau}, \boldsymbol{u}\right\rangle \mathrm{d} \tau \text { for a.e. } t \in\right] 0, T\right]
\end{aligned}
$$

By comparing this equality with (3.18) we then get

$$
\begin{aligned}
\frac{1}{2} \int_{\Omega}[\sigma(x, t) & \left.: B(x): \sigma(x, t)-\sigma^{0}(x): B(x): \sigma^{0}(x)\right] \mathrm{d} x \\
+\iint_{\Omega_{t}} \varphi(\sigma, x) \mathrm{d} x \mathrm{~d} \tau & +\iint_{\Omega_{t}} \varphi^{*}\left(\frac{\partial}{\partial \tau}(\varepsilon-B(x): \sigma), x\right) \mathrm{d} x \mathrm{~d} \tau \\
& \left.\left.\leqslant \iint_{\Omega_{t}} \sigma: \frac{\partial \varepsilon}{\partial t} \mathrm{~d} x \mathrm{~d} \tau \quad \text { for a.e. } t \in\right] 0, T\right]
\end{aligned}
$$

If $\sigma \in W^{1,1}\left(0, T ; L^{2}(\Omega)_{\mathrm{s}}^{9}\right)$, then

$$
\int_{\Omega}\left[\sigma(x, t): B(x): \sigma(x, t)-\sigma^{0}(x): B(x): \sigma^{0}(x)\right] \mathrm{d} x=\iint_{\Omega_{t}} \frac{\partial}{\partial t}(\sigma: B: \sigma) \mathrm{d} x \mathrm{~d} \tau,
$$

and the inequality (3.22) is equivalent to (2.16) a.e. in $\Omega_{T}$ (this is easily checked, for, by (2.12), the opposite inequality is automatically satisfied). In $\S 2$ we saw that (2.16) is equivalent to the inclusion (3.5).

In conclusion problem 3.1 is a weak formulation of the initial- and boundaryvalue problem (3.5)-(3.9). The extra-regularity on $\boldsymbol{u}$ and $\sigma$ that we assumed in this interpretation will be derived under suitable assumptions on the data (see remark 4.4 below).

\subsection{Elastoplasticity without strain-hardening}

For $\varphi=I_{K}$, the indicator function of a closed convex set $K \subset D_{\mathrm{s}}^{9}$ (the linear space of symmetric deviatoric tensors) containing the origin, the classical PrandtlReuss model is retrieved from (3.5). However, this corresponds to $p=\infty$ and $q=1$, a choice that we have so far excluded. Let us then see which modifications are needed if $\varphi$ has linear growth at infinity, as in the case of viscoplasticity. (In order to simplify this setting we assume that the Dirichlet part of the boundary, $\Gamma_{0}$, is empty.)

In this case the functional

$$
r \mapsto \iint_{\Omega_{T}} \varphi^{*}(r) \mathrm{d} x \mathrm{~d} t
$$

is just coercive over $L^{1}\left(\Omega_{T}\right)_{\mathrm{S}}^{9}$. As this space has no predual, by a standard procedure we embed it into the space of the bounded $\mathbb{R}_{\mathrm{S}}^{9}$-valued Radon measures over $\Omega_{T}$, 
which we may identify with $\left(C_{0}^{0}\left(\overline{\Omega_{T}}\right)_{\mathrm{s}}^{9}\right)^{\prime}$, namely the topological dual of the Banach space of continuous functions $\overline{\Omega_{T}} \rightarrow \mathbb{R}_{\mathrm{s}}^{9}$ that vanish at the space-time boundary. Setting $\xi:=\varepsilon-B(x): \sigma$, by (3.14) the inequality (3.18) implies that $\varphi^{*}(\partial \xi / \partial t, x)$ is a Radon measure, and (using the integral notation for the total variation)

$$
\iint_{\Omega_{T}} \varphi^{*}\left(\frac{\partial \xi}{\partial t}, x\right)<+\infty, \quad \text { whence } \frac{\partial \xi}{\partial t} \in\left(C^{0}\left(\overline{\Omega_{T}}\right)^{9}\right)^{\prime}
$$

provided that $\varphi$ is finite in a neighbourhood of the origin. In view of providing a more explicit representation of this functional, let us first decompose the measure $D_{t} \xi:=\partial \xi / \partial t$ as the sum of its absolutely continuous and singular parts with respect to the space-time Lebesgue measure: $D_{t} \xi=D_{t}^{(a)} \xi+D_{t}^{(\mathbf{s})} \xi$. Let us also denote by $\mathrm{d} D_{t}^{(\mathrm{s})} \xi / \mathrm{d}\left|D_{t}^{(\mathrm{s})} \xi\right|$ the Radon-Nikodým derivative of $D_{t}^{(\mathrm{s})} \xi$ with respect to its variation $\left|D_{t}^{(\mathrm{s})} \xi\right|$, and denote by $\varphi_{\infty}^{*}$ the asymptotic function of $\varphi^{*}$ :

$$
\left.\begin{array}{c}
\varphi_{\infty}^{*}(v, x):=\lim _{t \rightarrow+\infty} \frac{\varphi^{*}\left(v_{0}+t v, x\right)-\varphi^{*}\left(v_{0}, x\right)}{t} \\
D_{\mathrm{s}}^{9} \text {, for a.e. } x \in \Omega, \text { for all } v_{0} \in D_{\mathrm{s}}^{9} \text { such that } \varphi^{*}\left(v_{0}, x\right)<+\infty
\end{array}\right\}
$$

(this function does not depend on $v_{0}$ ). For example, for $\varphi=I_{K}, \varphi_{\infty}^{*}$ coincides with $\varphi^{*}=I_{K}^{*}$. Along the lines of a classical theory (see $\left.[8,25,45]\right)$, we can then represent the total variation as the sum of two genuine integrals:

$$
\begin{aligned}
\iint_{\Omega_{T}} \varphi^{*}\left(D_{t} \xi, x\right)=\iint_{\Omega_{T}} \varphi^{*}\left(D_{t}^{(a)} \xi, x\right) \mathrm{d} x \mathrm{~d} t & \\
& \quad+\iint_{\Omega_{T}} \varphi_{\infty}^{*}\left(\frac{D_{t}^{(\mathrm{s})} \xi}{\left|D_{t}^{(\mathrm{s})} \xi\right|}, x\right)\left|D_{t}^{(\mathrm{s})} \xi(\mathrm{d} x, \mathrm{~d} t)\right| .
\end{aligned}
$$

Here we replace $V$ by the space of functions with bounded deformation,

$$
\operatorname{BD}(\Omega):=\left\{\boldsymbol{w} \in L^{1}(\Omega)^{3}: \nabla^{\mathrm{s}} \boldsymbol{w} \in\left(C_{0}^{0}(\bar{\Omega})_{\mathrm{s}}^{9}\right)^{\prime}\right\},
$$

which is a Banach space equipped with the graph norm and has a separable predual (see, for example, $[7,88,89,97,98]$ ). Nevertheless, $\varepsilon$ may be singular not only in space but also in time; we then set

$$
X\left(\Omega_{T}\right):=\left\{\boldsymbol{\mu} \in\left(C_{0}^{0}\left(\overline{\Omega_{T}}\right)^{3}\right)^{\prime}: \nabla^{\mathrm{s}} \boldsymbol{\mu} \in\left(C_{0}^{0}\left(\overline{\Omega_{T}}\right)_{\mathrm{s}}^{9}\right)^{\prime}\right\},
$$

which is also a Banach space equipped with the graph norm, and assume that $\boldsymbol{u} \in$ $W^{1, \infty}\left(0, T ; L^{2}(\Omega)^{3}\right) \cap X\left(\Omega_{T}\right)$. Moreover, here we replace the regularity hypothesis $\boldsymbol{f}_{2} \in W^{1, p}\left(0, T ; V^{\prime}\right)$ in $(3.13)$ by $\boldsymbol{f}_{2} \in W_{w *}^{1, \infty}\left(0, T ; \operatorname{BD}(\Omega)^{\prime}\right)$. (Here the index $w *$ indicates that the vector-valued functions of this space are only assumed to be weakly-* measurable from $] 0, T\left[\right.$ to $\operatorname{BD}(\Omega)^{\prime}$ : this amendment is needed because the space $\operatorname{BD}(\Omega)$ is neither reflexive nor separable.)

Finally, we just outline the less simple case in which $\Gamma_{0}$ is non-empty. A source of difficulty arises by the fact that, although the trace on $\Gamma_{0}$ is meaningful in $\operatorname{BD}(\Omega)$, the Dirichlet condition (3.8) is not stable by weak-* convergence in this space (see $[7,88,89,97])$. This analytical difficulty has a mechanical counterpart, as plastic slip may occur on the Dirichlet part of the boundary. This phenomenon 
may be accounted for by assuming that $\nabla^{\mathrm{s}} \boldsymbol{u}$ is a Radon measure over $\Omega \cup \Gamma_{0}$, and then by requiring (instead of the Dirichlet condition) that

$$
\varepsilon_{i j}=-\frac{1}{2}\left(u_{i} \nu_{j}+u_{j} \nu_{i}\right) \mathcal{H}_{2} \text { on } \Gamma_{0 T} \text { for } i, j=1,2,3 \text {, }
$$

where by $\mathcal{H}_{2}$ we denote the bi-dimensional Hausdorff measure (see $[32,97]$ ).

\section{Analysis of the weak formulation}

In this section we prove the well-posedness of problem 3.1 for the nonlinear Maxwell model of viscoelasticity, and outline the extension to the Prandtl-Reuss model of elastoplasticity.

THEOREM 4.1 (existence). If (3.1)-(3.4), (3.12)-(3.14) are satisfied, then problem 3.1 has a solution.

Proof. For $p=q=2$ the procedure of $[13,14]$ might be applied. Here we use a different argument, which also provides uniform estimates that will be used in the homogenization procedure.

(i) Approximation. In view of introducing a time-discretized problem, let us fix any $m \in \mathbb{N}$ and set

$$
\left.\begin{array}{c}
h:=\frac{T}{m}, \quad \boldsymbol{f}_{m}^{n}:=\frac{1}{h} \int_{(n-1) h}^{n h} \boldsymbol{f}(\tau) \mathrm{d} \tau \in V^{\prime} \quad \text { for } n=1, \ldots, m, \\
\boldsymbol{u}_{m}^{0}:=\boldsymbol{u}^{0}, \quad \varepsilon_{m}^{0}:=\varepsilon^{0}\left(:=\nabla^{\mathrm{s}} \boldsymbol{u}^{0}\right), \quad \sigma_{m}^{0}:=\sigma^{0} \text { a.e. in } \Omega,
\end{array}\right\}
$$

A condition like (3.14) is thus satisfied with $v$ in place of $v_{(\mathrm{d})}$ :

$$
\left.\begin{array}{c}
\text { for all } m \in \mathbb{N}, \quad \exists a_{m}, b_{m}, \bar{a}, \bar{b}>0: \text { for all } v \in \mathbb{R}_{\mathrm{s}}^{9} \quad \text { for a.e. } x \in \Omega, \\
a_{m}|v|^{p}-b_{m} \leqslant \varphi_{m}(v, x) \leqslant \bar{a}|v|^{p}+\bar{b} .
\end{array}\right\}
$$

Problem 4.2. Find $\boldsymbol{u}_{m}^{n} \in V_{2}$ and $\sigma_{m}^{n} \in L^{p}(\Omega)_{\mathrm{s}}^{9}$ for $n=1, \ldots, m$ such that, setting $\boldsymbol{v}_{m}^{n}:=\left(\boldsymbol{u}_{m}^{n}-\boldsymbol{u}_{m}^{n-1}\right) / h$ and $\varepsilon_{m}^{n}:=\nabla^{\mathrm{s}} \boldsymbol{u}_{m}^{n}$,

$$
\begin{gathered}
\frac{\varepsilon_{m}^{n}-\varepsilon_{m}^{n-1}}{h}-B(x): \frac{\sigma_{m}^{n}-\sigma_{m}^{n-1}}{h} \in \partial \varphi_{m}\left(\sigma_{m}^{n}, x\right) \quad \text { a.e. in } \Omega, \\
\rho \frac{\boldsymbol{v}_{m}^{n}-\boldsymbol{v}_{m}^{n-1}}{h}-\nabla^{*} \cdot \sigma_{m}^{n}=\boldsymbol{f}_{m}^{n} \quad \text { in } V^{\prime} .
\end{gathered}
$$

We prove the existence of a solution of this problem step by step. For any integer $n \geqslant 1$ and a.e. $x \in \Omega$ the function

$$
\psi_{m}^{n}(s, x):=h \varphi_{m}(s, x)+\frac{1}{2} s: B(x): s+\left(\varepsilon_{m}^{n-1}-B(x): \sigma_{m}^{n-1}\right): s \quad \text { for all } s \in \mathbb{R}_{\mathrm{s}}^{9}
$$

is lower semicontinuous and convex. We denote its convex conjugate by $\left(\psi_{m}^{n}\right)^{*}(\cdot, x)$. The inclusion (4.4) also reads $\nabla^{\mathrm{s}} \boldsymbol{u}_{m}^{n} \in \partial \psi_{m}^{n}\left(\sigma_{m}^{n}, x\right)$, namely $\sigma_{m}^{n} \in \partial\left(\psi_{m}^{n}\right)^{*}\left(\nabla^{\mathrm{s}} \boldsymbol{u}_{m}^{n}, x\right)$ 
a.e. in $\Omega$. Equation (4.5) is thus the Euler equation for the minimization of a functional of the form

$$
J_{m}^{n}(\boldsymbol{u}):=\int_{\Omega}\left[\frac{1}{2} \rho|\boldsymbol{u}|^{2}+h^{2}\left(\psi_{m}^{n}\right)^{*}\left(\nabla^{\mathrm{s}} \boldsymbol{u}, x\right)\right] \mathrm{d} x-\left\langle\boldsymbol{\eta}_{m}^{n}, \boldsymbol{u}\right\rangle \quad \text { for all } \boldsymbol{u} \in V_{2},
$$

with $\boldsymbol{\eta}_{m}^{n}$ dependent on $\boldsymbol{f}_{m}^{n}, \boldsymbol{u}^{0}, \boldsymbol{v}^{0}$ and $h$. The functional $J_{m}^{n}: V_{2} \rightarrow \mathbb{R} \cup\{+\infty\}$ is lower semicontinuous and (strictly) convex, and by (4.3) it is coercive over $V_{2}$. The system (4.4), (4.5) has then one (and only one) solution, $\boldsymbol{u}_{m}^{n} \in V_{2}$, which determines $\varepsilon_{m}^{n} \in L^{2}(\Omega)_{\mathrm{s}}^{9}$. Because of (3.2) and (4.3), the mapping $\sigma \mapsto B \sigma+h \partial \varphi(\sigma)$ is coercive and injective; hence, it is invertible. By (4.4) $\varepsilon_{m}^{n}$ then determines $\sigma_{m}^{n} \in L^{p}(\Omega)_{\mathrm{s}}^{9}$.

By (2.9) and (3.4) the inclusion (4.4) also yields $\varepsilon_{m(\mathrm{~s})}^{n}-\varepsilon_{m(\mathrm{~s})}^{n-1}=\beta\left(\sigma_{m(\mathrm{~s})}^{n}-\sigma_{m(\mathrm{~s})}^{n-1}\right)$ a.e. in $\Omega$ for any $n$, whence by (3.12)

$$
\varepsilon_{m(\mathrm{~s})}^{n}=\beta \sigma_{m(\mathrm{~s})}^{n} \quad \text { a.e. in } \Omega \text { for all } n .
$$

For any family $\left\{\phi_{m}^{n}\right\}_{n=0, \ldots, m}$ of functions $\Omega \rightarrow \mathbb{R}$, let us set

$$
\left.\begin{array}{rl}
\phi_{m} & :=\text { piecewise linear time-interpolate of } \phi_{m}^{0}, \ldots, \phi_{m}^{m} \text { a.e. in } \Omega, \\
\bar{\phi}_{m}(\cdot, t) & \left.:=\phi_{m}^{n} \quad \text { a.e. in } \Omega \text { for all } t \in\right](n-1) h, n h[\text { for } n=1, \ldots, m .
\end{array}\right\}
$$

Equations (4.4) and (4.5) then read

$$
\begin{gathered}
\frac{\partial \varepsilon_{m}}{\partial t}-B(x): \frac{\partial \sigma_{m}}{\partial t} \in \partial \varphi_{m}\left(\bar{\sigma}_{m}, x\right) \quad \text { a.e. in } \Omega_{T} \\
\left.\rho \frac{\partial \boldsymbol{v}_{m}}{\partial t}-\nabla^{*} \cdot \bar{\sigma}_{m}=\overline{\boldsymbol{f}}_{m} \quad \text { in } V^{\prime}, \text { a.e. in }\right] 0, T[
\end{gathered}
$$

(ii) Energy estimates. Multiplying (4.10) by $\boldsymbol{v}_{m}$ and then integrating over $\Omega$ we get

$$
\left.\frac{1}{2} \frac{\mathrm{d}}{\mathrm{d} t} \int_{\Omega} \rho\left|\boldsymbol{v}_{m}\right|^{2} \mathrm{~d} x+\int_{\Omega} \bar{\sigma}_{m}: \frac{\partial \varepsilon_{m}}{\partial t} \mathrm{~d} x=\left\langle\overline{\boldsymbol{f}}_{m}, \boldsymbol{v}_{m}\right\rangle \quad \text { a.e. in }\right] 0, T[.
$$

By the Fenchel property (2.13), (4.9) is equivalent to

$$
\begin{aligned}
\varphi_{m}\left(\bar{\sigma}_{m}, x\right)+\varphi_{m}^{*}\left(\frac{\partial \varepsilon_{m}}{\partial \tau}-\right. & \left.B(x): \frac{\partial \sigma_{m}}{\partial \tau}, x\right) \\
= & \frac{\partial \varepsilon_{m}}{\partial t}: \bar{\sigma}_{m}-\bar{\sigma}_{m}: B(x): \frac{\partial \sigma_{m}}{\partial t} \quad \text { a.e. in } \Omega_{T} .
\end{aligned}
$$

The two latter equalities yield

$$
\begin{gathered}
\frac{1}{2} \int_{\Omega}\left(\rho\left|\boldsymbol{v}_{m}\right|^{2}+\sigma_{m}: B(x): \sigma_{m}\right)(\cdot, t) \mathrm{d} x-\frac{1}{2} \int_{\Omega}\left(\rho\left|\boldsymbol{v}^{0}\right|^{2}+\sigma^{0}: B(x): \sigma^{0}\right) \mathrm{d} x \\
\quad+\iint_{\Omega_{t}}\left[\varphi_{m}\left(\bar{\sigma}_{m}, x\right)+\varphi_{m}^{*}\left(\frac{\partial}{\partial \tau}\left(\varepsilon_{m}-B(x): \sigma_{m}\right), x\right)\right] \mathrm{d} x \mathrm{~d} \tau \\
=\iint_{\Omega_{t}} \overline{\boldsymbol{f}}_{1} \cdot \frac{\partial \boldsymbol{u}_{m}}{\partial \tau} \mathrm{d} x \mathrm{~d} \tau+\left\langle\overline{\boldsymbol{f}}_{2}(t), \overline{\boldsymbol{u}}_{m}(\cdot, t)\right\rangle \\
\left.-\left\langle\boldsymbol{f}_{2}(0), \boldsymbol{u}^{0}\right\rangle-\int_{0}^{t}\left\langle\frac{\partial \boldsymbol{f}_{2}}{\partial \tau}, \overline{\boldsymbol{u}}_{m}\right\rangle \mathrm{d} \tau \quad \text { for all } t \in\right] 0, T[
\end{gathered}
$$


Henceforth, we shall denote by $C_{i}$ suitable positive constants that may depend on the data. Recalling (3.1), (3.2), (3.12)-(3.15), the energy equality (4.13) yields (denoting by $|\Omega|$ the volume of $\Omega$ )

$$
\begin{gathered}
\frac{1}{2} \int_{\Omega}\left(\rho\left|\boldsymbol{v}_{m}\right|^{2}+\bar{c}\left|\sigma_{m}\right|^{2}\right)(x, t) \mathrm{d} x+\iint_{\Omega_{t}}\left(a\left|\sigma_{m(\mathrm{~d})}\right|^{p}+c\left|\frac{\partial}{\partial \tau}\left(\varepsilon_{m}-B(x): \sigma_{m}\right)\right|^{q}\right) \mathrm{d} x \mathrm{~d} \tau \\
\leqslant \frac{1}{2} \int_{\Omega}\left(\rho\left|\boldsymbol{v}^{0}\right|^{2}+\sigma^{0}: B(x): \sigma^{0}\right)(x) \mathrm{d} x+\left\|\boldsymbol{f}_{1}\right\|_{L^{1}\left(0, t ; L^{2}(\Omega)^{3}\right)} \max _{[0, t]}\left\|\boldsymbol{v}_{m}\right\|_{L^{2}(\Omega)^{3}} \\
\left.\quad+C_{1}\left\|\boldsymbol{f}_{2}\right\|_{W^{1, p}\left(0, t ; V^{\prime}\right)}\left\|\boldsymbol{u}_{m}\right\|_{L^{q}(0, t ; V)}+(b+d) t|\Omega| \quad \text { for all } t \in\right] 0, T[. \quad \text { (4.14) }
\end{gathered}
$$

By the Korn inequality and recalling that $q \leqslant 2$,

$$
\begin{aligned}
\left\|\boldsymbol{u}_{m}\right\|_{V} & \leqslant C_{2}\left\|\varepsilon_{m}\right\|_{L^{q}(\Omega)^{9}} \\
& \left.\leqslant C_{2}\left\|\varepsilon_{m}-B(x): \sigma_{m}\right\|_{L^{q}(\Omega)^{9}}+C_{3}\left\|\sigma_{m}\right\|_{L^{2}(\Omega)^{9}} \quad \text { for all } t \in\right] 0, T[.
\end{aligned}
$$

By (4.14) a standard computation then yields

$$
\begin{aligned}
& \left\|\boldsymbol{u}_{m}\right\|_{W^{1, \infty}\left(0, T ; L^{2}(\Omega)^{3}\right) \cap L^{q}(0, T ; V)},\left\|\sigma_{m}\right\|_{L^{\infty}\left(0, T ; L^{2}(\Omega)^{9}\right)}, \\
& \qquad \sigma_{m(\mathrm{~d})}\left\|_{L^{p}\left(\Omega_{T}\right)^{9}},\right\| \frac{\partial}{\partial t}\left(\varepsilon_{m}-B(x): \sigma_{m}\right) \|_{L^{q}\left(\Omega_{T}\right)^{9}} \leqslant C_{4} .
\end{aligned}
$$

(iii) Passage to the limit. By the estimates (4.15) there exist $\boldsymbol{u}, \sigma$ such that, possibly taking $m \rightarrow \infty$ along a subsequence,

$$
\begin{aligned}
\boldsymbol{u}_{m} & \stackrel{*}{\rightarrow} \boldsymbol{u} \quad \text { in } W^{1, \infty}\left(0, T ; L^{2}(\Omega)^{3}\right) \cap L^{q}(0, T ; V), \\
\sigma_{m} & \stackrel{*}{\rightarrow} \sigma \quad \text { in } L^{\infty}\left(0, T ; L^{2}(\Omega)^{9}\right), \\
\sigma_{m(\mathrm{~d})} & \rightarrow \sigma_{(\mathrm{d})} \quad \text { in } L^{p}\left(\Omega_{T}\right)^{9}, \\
\frac{\partial}{\partial t}\left(\varepsilon_{m}-B(x): \sigma_{m}\right) & \rightarrow \frac{\partial}{\partial t}(\varepsilon-B(x): \sigma) \quad \text { in } L^{q}\left(\Omega_{T}\right)^{9},
\end{aligned}
$$

where by ' $\rightarrow$ ', ' $\rightarrow$ ', ' $\stackrel{*}{\rightarrow}$ we denote the strong, weak and weak-* convergence, respectively.

By passing to the limit in (4.10) and to the inferior limit in (4.13), we then get (3.18) and (3.19).

Theorem 4.3 (regularity). Let (3.1)-(3.4), (3.12)-(3.14) be satisfied and

$$
\begin{gathered}
\boldsymbol{v}^{0} \in L^{2}(\Omega)^{3} \cap V, \\
\partial \varphi\left(\sigma^{0}, x\right) \cap L^{2}(\Omega)_{\mathrm{s}}^{9} \neq \emptyset, \quad \boldsymbol{f}(0)+\nabla^{*} \cdot \sigma^{0} \in L^{2}(\Omega)^{3}, \\
\boldsymbol{f}_{1} \in W^{1,1}\left(0, T ; L^{2}(\Omega)^{3}\right), \quad \boldsymbol{f}_{2} \in W^{2, p}\left(0, T ; V^{\prime}\right) .
\end{gathered}
$$

Then problem 3.1 has a solution such that, besides the regularity (3.16) and (3.17),

$$
\boldsymbol{u} \in W^{2, \infty}\left(0, T ; L^{2}(\Omega)^{3}\right) \cap W^{1, q}(0, T ; V), \quad \sigma \in W^{1, \infty}\left(0, T ; L^{2}(\Omega)^{9}\right) .
$$

Proof. In view of deriving further a priori estimates, first let us set $\boldsymbol{u}_{m}^{-1}:=\boldsymbol{u}^{0}-h \boldsymbol{v}^{0}$ a.e. in $\Omega$, and note that $\varepsilon_{m}^{-1}:=\nabla^{\mathrm{s}} \boldsymbol{u}_{m}^{-1}=\nabla^{\mathrm{s}}\left(\boldsymbol{u}^{0}-h \boldsymbol{v}^{0}\right)$ in $L^{2}(\Omega)_{\mathrm{s}}^{9}($ see $(3.12)$ 
and (4.20)). By (4.21) there exist $\sigma_{m}^{-1} \in L^{2}(\Omega)_{\mathrm{s}}^{9}$ and $\boldsymbol{v}_{m}^{-1} \in L^{2}(\Omega)^{3}$ such that (4.4) and (4.5) are also satisfied for $n=0$. Moreover,

$$
\left\|\frac{\sigma_{m}^{0}-\sigma_{m}^{-1}}{h}\right\|_{L^{2}(\Omega)^{9}},\left\|\frac{\boldsymbol{v}_{m}^{0}-\boldsymbol{v}_{m}^{-1}}{h}\right\|_{L^{2}(\Omega)^{3}} \leqslant C_{5} .
$$

Let us denote the right-hand side of (4.4) by $\xi_{m}^{n}$ and set

$$
\begin{aligned}
& e_{m}^{n}:=\frac{\varepsilon_{m}^{n}-\varepsilon_{m}^{n-1}}{h}, \quad s_{m}^{n}:=\frac{\sigma_{m}^{n}-\sigma_{m}^{n-1}}{h}, \quad \boldsymbol{w}_{m}^{n}:=\frac{\boldsymbol{v}_{m}^{n}-\boldsymbol{v}_{m}^{n-1}}{h} \\
& \text { a.e. in } \Omega \text { for } n=0, \ldots, m \text {. }
\end{aligned}
$$

Taking the incremental ratios of (4.4) and (4.5) for $n=1, \ldots, m$, we obtain

$$
\begin{gathered}
\frac{e_{m}^{n}-e_{m}^{n-1}}{h}-B(x): \frac{s_{m}^{n}-s_{m}^{n-1}}{h}=\frac{\xi_{m}^{n}-\xi_{m}^{n-1}}{h} \text { a.e. in } \Omega, \\
\rho \frac{\boldsymbol{w}_{m}^{n}-\boldsymbol{w}_{m}^{n-1}}{h}-\nabla^{*} \cdot s_{m}^{n}=\frac{\boldsymbol{f}_{m}^{n}-\boldsymbol{f}_{m}^{n-1}}{h} \text { in } V^{\prime} .
\end{gathered}
$$

Multiplying these equations respectively by $s_{m}^{n}$ and $\boldsymbol{w}_{m}^{n}$, by the monotonicity of $\partial \varphi_{m}(\cdot, x)$ we get

$$
\begin{aligned}
& \frac{1}{2} \int_{\Omega}\left[\left(\rho\left|\overline{\boldsymbol{w}}_{m}\right|^{2}+\bar{s}_{m}: B(x): \bar{s}_{m}\right)(\cdot, t)-\left(\rho\left|\boldsymbol{w}^{0}\right|^{2}+s^{0}: B(x): s^{0}\right)\right] \mathrm{d} x \\
& \leqslant \int_{0}^{t}\left\langle\frac{\partial \boldsymbol{f}_{m}}{\partial \tau}, \overline{\boldsymbol{w}}_{m}\right\rangle \mathrm{d} \tau \\
& \leqslant\left\|\boldsymbol{f}_{1}\right\|_{W^{1,1}\left(0, T ; L^{2}(\Omega)^{3}\right)} \max _{[0, t]}\left\|\boldsymbol{w}_{m}\right\|_{L^{2}(\Omega)^{3}} \\
& \left.\quad+C_{6}\left\|\boldsymbol{f}_{2}\right\|_{W^{2, p}\left(0, T ; V^{\prime}\right)}\left\|\boldsymbol{v}_{m}\right\|_{L^{q}(0, t ; V)} \quad \text { for all } t \in\right] 0, T[.
\end{aligned}
$$

As above, by the Korn inequality and as $q \leqslant 2$,

$$
\begin{aligned}
\left\|\boldsymbol{v}_{m}\right\|_{V} & \leqslant C_{7}\left\|e_{m}\right\|_{L^{q}(\Omega)^{9}} \\
& \left.\leqslant C_{7}\left\|e_{m}-B(x): s_{m}\right\|_{L^{q}(\Omega)^{9}}+C_{8}\left\|s_{m}\right\|_{L^{2}(\Omega)^{9}} \quad \text { for all } t \in\right] 0, T[.
\end{aligned}
$$

By (4.15) and (4.24)-(4.26) a standard computation then yields

$$
\left\|\boldsymbol{u}_{m}\right\|_{W^{2, \infty}\left(0, T ; L^{2}(\Omega)^{3}\right)},\left\|\sigma_{m}\right\|_{W^{1, \infty}\left(0, T ; L^{2}(\Omega)^{9}\right)} \leqslant C_{9} .
$$

As by (4.15) $\partial\left(\varepsilon_{m}-B(x): \sigma_{m}\right) / \partial t$ is uniformly bounded in $L^{q}\left(\Omega_{T}\right)^{9}$, we then get

$$
\left\|u_{m}\right\|_{W^{1, q}(0, T ; V)} \leqslant C_{2}\left\|\varepsilon_{m}\right\|_{W^{1, q}\left(0, T ; L^{q}(\Omega)^{9}\right)} \leqslant C_{10} .
$$

These uniform estimates yield (4.23).

REMARK 4.4.

(i) If $\boldsymbol{f}_{2}$ is of the form (3.21), we infer from (3.20) and (4.23) that $\nabla \cdot \sigma \in$ $L_{\text {loc }}^{2}\left(\Omega_{T}\right)^{3}$ and then equation (3.6) is satisfied a.e. in $\Omega_{T}$.

(ii) If, moreover, $\boldsymbol{f}_{2} \equiv 0$, then both $\partial^{2} \boldsymbol{u} / \partial t^{2}$ and $\nabla \cdot \sigma$ are elements of $L^{2}\left(\Omega_{T}\right)^{3}$, so that we may multiply (3.20) by $\partial \boldsymbol{u} / \partial t \in L^{2}\left(\Omega_{T}\right)^{3}$. As we saw in $\S 3$, the inequality (3.18) is then rigorously equivalent to the inclusion (3.5), so that in this case problem 3.1 is fully justified and $(\boldsymbol{u}, \sigma)$ is a strong solution. 
Theorem 4.5 (well-posedness). For $i=1,2$ let $\left(\boldsymbol{u}^{0 i}, \boldsymbol{v}^{0 i}, \sigma^{0 i}, \boldsymbol{f}_{1}^{i}\right)$ satisfy the hypotheses of theorem 4.3 and let $\boldsymbol{f}_{2}^{i} \equiv 0$. Let $\left(\boldsymbol{u}^{i}, \sigma^{i}\right)$ be two corresponding solutions of problem 3.1 that satisfy (4.23). Then there exists a constant $L>0$ independent of the data such that, setting $\tilde{\boldsymbol{u}}:=\boldsymbol{u}^{1}-\boldsymbol{u}^{2}$ and defining $\tilde{\sigma}, \tilde{\boldsymbol{f}}$ and so on similarly, we obtain

$$
\begin{aligned}
& \left\|\frac{\partial \tilde{\boldsymbol{u}}}{\partial t}\right\|_{W^{1, \infty}\left(0, T ; L^{2}(\Omega)^{3}\right)}+\|\tilde{\sigma}\|_{L^{\infty}\left(0, T ; L^{2}(\Omega)^{9}\right)} \\
& \quad \leqslant L\left(\left\|\tilde{\boldsymbol{v}}^{0}\right\|_{L^{2}(\Omega)^{3}}+\left\|\tilde{\sigma}^{0}\right\|_{L^{2}(\Omega)^{9}}+\left\|\tilde{\boldsymbol{f}}_{1}\right\|_{W^{1,1}\left(0, T ; L^{2}(\Omega)^{3}\right)}+\left\|\tilde{\boldsymbol{f}}_{2}\right\|_{W^{2,1}\left(0, T ; V^{\prime}\right)}\right) .
\end{aligned}
$$

In particular problem 3.1 has just one solution that satisfies (4.23).

Proof. Let us write (3.5) and (3.20) for $i=1,2$, and multiply them by $\tilde{\sigma}$ and $\partial \tilde{\boldsymbol{u}} / \partial t$ respectively; this is admissible because of the regularity (4.23). By combining these formulae we get

$$
\begin{aligned}
& \frac{1}{2} \int_{\Omega}\left(\rho\left|\frac{\partial \tilde{\boldsymbol{u}}}{\partial t}\right|^{2}+\tilde{\sigma}: B(x): \tilde{\sigma}\right)(\cdot, t) \mathrm{d} x-\frac{1}{2} \int_{\Omega}\left(\rho\left|\tilde{\boldsymbol{v}}^{0}\right|^{2}+\tilde{\sigma}^{0}: B(x): \tilde{\sigma}^{0}\right) \mathrm{d} x \\
& \quad \leqslant\left\|\tilde{\boldsymbol{f}}_{1}\right\|_{W^{1,1}\left(0, T ; L^{2}(\Omega)^{3}\right)}\left\|\frac{\partial \tilde{\boldsymbol{u}}}{\partial \tau}\right\|_{L^{\infty}\left(0, t ; L^{2}(\Omega)^{3}\right)}+\left\|\tilde{\boldsymbol{f}}_{2}\right\|_{W^{2,1}\left(0, T ; V^{\prime}\right)}\|\tilde{\boldsymbol{u}}\|_{L^{\infty}(0, t ; V)}
\end{aligned}
$$

for all $t \in] 0, T]$,

whence (4.29) follows. The final statement is straightforward.

\subsection{Limit cases}

In several cases the inertia term is so small that it may be neglected. As $\rho \rightarrow 0$ in $L^{\infty}(\Omega)$, a subsequence of solutions of problem 3.1 converges to a solution of the reduced problem, in which the equation of continuum dynamics (3.20) is replaced by the quasi-static force-balance equation

$$
\left.-\nabla^{*} \cdot \sigma=f \text { in } V^{\prime} \text { a.e. in }\right] 0, T[.
$$

If $\boldsymbol{f} \in W^{1,1}\left(0, T ; V^{\prime}\right)$, then in place of (3.16) here we merely obtain the regularity

$$
\boldsymbol{u} \in L^{q}(0, T ; V), \quad \sigma \in L^{\infty}\left(0, T ; L^{2}(\Omega)_{\mathrm{s}}^{9}\right) .
$$

No difficulty arises in passing to the limit as $B \rightarrow 0$, although the regularity $\sigma \in L^{\infty}\left(0, T ; L^{2}(\Omega)_{\mathrm{s}}^{9}\right)$ is then lost.

As we saw, the Prandtl-Reuss model corresponds to $\varphi=I_{K}, K$ being a closed convex subset of $D_{\mathrm{s}}^{9}$ that contains the origin, and in this case the Dirichlet boundary condition (3.8) is lost. We may approximate $\varphi=I_{K}$ by a sequence of penalized functions $\left\{\varphi_{n}\right\}$ that satisfy the hypotheses of theorem 4.1 (e.g. via Yosida approximation), so that for any $n$ this problem has a solution $\left(\boldsymbol{u}_{n}, \sigma_{n}\right)$. If $\boldsymbol{f}_{2} \in$ $W_{w *}^{1, \infty}\left(0, T ; \mathrm{BD}(\Omega)^{\prime}\right)$, by a compactness and lower semicontinuity argument one can easily see that a subsequence of solutions converges to a solution of the limit problem such that

$$
\boldsymbol{u} \in W^{1, \infty}\left(0, T ; L^{2}(\Omega)^{3}\right), \quad \sigma \in L^{\infty}\left(\Omega_{T}\right)_{\mathrm{s}}^{9}, \quad \varepsilon, \frac{\partial}{\partial t}(\varepsilon-B(x): \sigma) \in\left(C^{0}\left(\overline{\Omega_{T}}\right)_{\mathrm{s}}^{9}\right)^{\prime} ;
$$


(see, for example, $[11,98]$ ). At this point one may also pass to the limit as $\rho \rightarrow 0$ in $L^{\infty}(\Omega)$. Some caution is needed in the mechanical interpretation of this setting, for the hypothesis of small displacements may fail due to perfect plastic flow. In the absence of a safe load condition, mechanical failure might also occur (see, for example, $[29,93,97])$.

One may also pass to the limit as $\varphi(\cdot, x) \rightarrow 0$ locally uniformly in $\mathbb{R}_{\mathrm{s}}^{9}$, a.e. in $\Omega$, e.g. if $\varphi(0, x)=0$, one may set $\varphi_{n}(v, x)=\varphi(v / n, x)$, and then take $n \rightarrow \infty$. One thus retrieves the linear elastic law $\varepsilon=B(x): \sigma$, with the reduced regularity $\boldsymbol{u} \in W^{1, \infty}\left(0, T ; L^{2}(\Omega)_{\mathrm{s}}^{9}\right) \cap L^{\infty}\left(0, T ; V_{2}\right), \sigma \in L^{\infty}\left(0, T ; L^{2}(\Omega)_{\mathrm{s}}^{9}\right)$.

More generally the above limit behaviours may occur in different parts of $\Omega$. For instance, our material may be purely viscous $(B(x)=0)$ a.e. in $\tilde{\Omega}$ and purely elastic $(\varphi(\cdot, x)=0)$ a.e. in $\Omega \backslash \tilde{\Omega}$.

The regularity theorem (theorem 4.3) is also easily extended to this setting, with the natural amendments due to the exceptional exponent $q=1$. For instance, here it is in order to assume that $\boldsymbol{f}_{2} \in W_{w *}^{2,1}\left(0, T ; \mathrm{BD}(\Omega)^{\prime}\right)$, and the enhanced regularity for $\boldsymbol{u}$ reads (see (3.27))

$$
\boldsymbol{u} \in W^{2, \infty}\left(0, T ; L^{2}(\Omega)^{3}\right), \quad \frac{\partial \boldsymbol{u}}{\partial t} \in X\left(\Omega_{T}\right) .
$$

On the other hand, difficulties arise in extending the well-posedness theorem (theorem 4.5), for in general the stress field $\sigma$ may not be expected to be continuous, and so (3.5) cannot be multiplied by $\sigma$.

\section{Two-scale homogenization}

In this section we assume that the function $\varphi$ and the tensor $B$ oscillate in space periodically, and we derive a two-length-scale model by passing to the limit in problem 3.1 as the period vanishes, via Nguetseng's notion of two-scale convergence $[4,80]$. We then illustrate how this result may be extended to the Prandtl-Reuss model.

\subsection{Two-length-scale model}

So far we have dealt with a single spatial variable, $x$. Here we regard $x$ as a coarse-length-scale variable, and also introduce a fine-length-scale variable $y$ that we let range through a reference volume element $Y:=\left[0,1\left[^{3}\right.\right.$. We identify $Y$ with

the unit torus $\mathcal{Y}$ and note that any $Y$-periodic function on $\mathbb{R}^{3}$ may thus be identified with a function on $\mathcal{Y}$. We assume that

$$
\begin{gathered}
\rho \in L^{\infty}(\Omega), \quad \rho \geqslant \rho_{0}: \text { const. }>0 \text { a.e. in } \Omega \\
B \in L^{\infty}(\mathcal{Y} ; \mathcal{M}), \quad \exists \bar{c}>0: \text { for all } v \in \mathbb{R}_{\mathrm{s}}^{9}, v: B(y): v \geqslant \bar{c}|v|^{2} \text { for a.e. } y \in \mathcal{Y} \\
\varphi: \mathbb{R}_{\mathrm{s}}^{9} \times \mathcal{Y} \rightarrow \mathbb{R} \cup\{+\infty\} \text { satisfies }(3.3),(3.4),(3.14) \text { with } \mathcal{Y} \text { in place of } \Omega
\end{gathered}
$$

We then set the constitutive law (3.5) in two-scale form:

$$
\frac{\partial \varepsilon}{\partial t}(x, y, t)-B(y): \frac{\partial \sigma}{\partial t}(x, y, t) \in \partial \varphi(\sigma(x, y, t), y) \quad \text { for a.e. }(x, y, t) \in \Omega_{T} \times \mathcal{Y} .
$$




\subsection{Two-scale weak formulation}

Dealing with functions of $x$ and $y$, we shall denote the gradient operators with respect to $x$ and $y$ by $\nabla_{x}$ and $\nabla_{y}$, respectively, and the corresponding symmetrized gradients by $\nabla_{x}^{\mathrm{s}}$ and $\nabla_{y}^{\mathrm{s}}$. For any function $w=w(y)$ we define the average and fluctuating components:

$$
\hat{w}:=\int_{\mathcal{Y}} w(y) \mathrm{d} y, \quad \tilde{w}:=w-\hat{w} \quad \text { for all } w \in L^{1}(\mathcal{Y}) .
$$

For technical reasons here we assume that $\boldsymbol{f}_{2} \equiv \mathbf{0}$ (i.e. $\boldsymbol{f} \equiv \boldsymbol{f}_{1}$ ). We also assume that we are given the following functions:

$$
\begin{gathered}
\boldsymbol{u}^{0} \in L^{2}(\Omega)^{3} \cap V, \quad \boldsymbol{u}_{(1)}^{0} \in L^{q}\left(\Omega ; W^{1, q}(\mathcal{Y})^{3}\right), \quad \boldsymbol{v}^{0} \in L^{2}(\Omega)^{3}, \quad \sigma^{0} \in L^{2}(\Omega \times \mathcal{Y})_{\mathrm{s}}^{9}, \\
\boldsymbol{f} \in L^{1}\left(0, T ; L^{2}(\Omega)^{3}\right) .
\end{gathered}
$$

We then set $\varepsilon^{0}:=\nabla^{\mathrm{s}} \boldsymbol{u}^{0}+\nabla_{y}^{\mathrm{s}} \boldsymbol{u}_{(1)}^{0}\left(\in L^{q}(\Omega \times \mathcal{Y})^{9}\right)$ and require that $\varepsilon_{(\mathrm{s})}^{0}=\beta \sigma_{(\mathrm{s})}^{0}$ a.e. in $\Omega \times \mathcal{Y}$.

Problem 5.1 (two-scale weak formulation). Find $(\boldsymbol{u}, \varepsilon, \sigma)$ such that

$$
\begin{aligned}
& \boldsymbol{u} \in W^{1, \infty}\left(0, T ; L^{2}(\Omega)^{3}\right), \quad \sigma \in L^{\infty}\left(0, T ; L^{2}(\Omega \times \mathcal{Y})_{\mathrm{s}}^{9}\right), \\
& \left.\sigma_{(\mathrm{d})} \in L^{p}\left(\Omega_{T} \times \mathcal{Y}\right)_{\mathrm{s}}^{9}, \quad \varepsilon, \frac{\partial}{\partial t}(\varepsilon-B(y): \sigma) \in L^{q}\left(\Omega_{T} \times \mathcal{Y}\right)_{\mathrm{s}}^{9},\right\} \\
& \exists \boldsymbol{u}_{(1)} \in L^{q}\left(\Omega_{T} ; W^{1, q}(\mathcal{Y})^{3}\right): \varepsilon=\nabla^{\mathrm{s}} \boldsymbol{u}+\nabla_{y}^{\mathrm{s}} \boldsymbol{u}_{(1)} \quad \text { a.e. in } \Omega_{T} \times \mathcal{Y}, \\
& \frac{1}{2} \int_{\Omega} \rho\left(\left|\frac{\partial \boldsymbol{u}}{\partial t}(x, t)\right|^{2}-\rho\left|\boldsymbol{v}^{0}\right|^{2}\right) \mathrm{d} x \\
& +\frac{1}{2} \iint_{\Omega \times \mathcal{Y}}\left(\sigma(x, t): B(y): \sigma(x, t)-\sigma^{0}: B(y): \sigma^{0}\right) \mathrm{d} x \mathrm{~d} y \\
& +\iiint_{\Omega_{t} \times \mathcal{Y}}\left[\varphi(\sigma, y)+\varphi^{*}\left(\frac{\partial}{\partial \tau}(\varepsilon-B(y): \sigma), y\right)\right] \mathrm{d} x \mathrm{~d} y \mathrm{~d} \tau \\
& \left.\left.\leqslant \iint_{\Omega_{t}} \boldsymbol{f} \cdot \frac{\partial \boldsymbol{u}}{\partial \tau} \mathrm{d} x \mathrm{~d} \tau \quad \text { for a.e. } t \in\right] 0, T\right] \\
& \iint_{\Omega_{T}}\left(\rho\left(\boldsymbol{u}^{0}-\boldsymbol{u}\right) \cdot \frac{\partial \boldsymbol{w}}{\partial t}+\int_{0}^{t} \hat{\sigma}(\cdot, \tau) \mathrm{d} \tau: \nabla \boldsymbol{w}\right) \mathrm{d} x \mathrm{~d} t \\
& =\iint_{\Omega_{T}}\left(\int_{0}^{t} \boldsymbol{f}(\cdot, \tau) \mathrm{d} \tau+\rho \hat{\boldsymbol{v}}^{0}\right) \cdot \boldsymbol{w} \mathrm{d} x \mathrm{~d} t \\
& \text { for all } \boldsymbol{w} \in H^{1}(0, T ; V), \boldsymbol{w}(\cdot, T)=\mathbf{0} \text { a.e. in } \Omega, \\
& \nabla_{y} \cdot \sigma=\mathbf{0} \text { in } \mathcal{D}^{\prime}(\mathcal{Y})^{3} \text { a.e. in } \Omega_{T} \text {. }
\end{aligned}
$$

\subsection{Interpretation}

In $\S 3$ we saw that (5.11) yields equation (3.20) and the initial conditions (3.7). Next we derive the two-scale constitutive law (5.4) from the inequality (5.10), 
assuming that $\nabla \cdot \sigma \in L^{2}\left(\Omega_{T}\right)^{3}$, so that equation (3.20) is satisfied a.e. in $\Omega_{T}$. Under further assumptions in theorem 5.6 we shall retrieve this regularity, and thus justify this interpretation. Multiplying (3.20) by $\partial \boldsymbol{u} / \partial t$ and integrating over $\Omega$ we get

$$
\frac{1}{2} \int_{\Omega} \rho\left(\left|\frac{\partial \boldsymbol{u}}{\partial t}(\cdot, t)\right|^{2}-\left|\boldsymbol{v}^{0}\right|^{2}\right) \mathrm{d} x+\iint_{\Omega_{t}} \hat{\sigma}: \frac{\partial \hat{\varepsilon}}{\partial \tau} \mathrm{d} x \mathrm{~d} \tau=\iint_{\Omega_{t}} \boldsymbol{f} \cdot \frac{\partial \boldsymbol{u}}{\partial \tau} \mathrm{d} x \mathrm{~d} \tau
$$

for a.e. $t \in] 0, T[$. On the other hand, by (5.9) and (5.12) we have

$$
\int_{\mathcal{Y}} \sigma: \frac{\partial \varepsilon}{\partial \tau} \mathrm{d} y=\int_{\mathcal{Y}} \sigma: \frac{\partial \hat{\varepsilon}}{\partial \tau} \mathrm{d} y+\int_{\mathcal{Y}} \sigma: \frac{\partial}{\partial \tau} \nabla_{y} \boldsymbol{u}_{(1)} \mathrm{d} y=\hat{\sigma}: \frac{\partial \hat{\varepsilon}}{\partial \tau} \quad \text { a.e. in } \Omega_{t}
$$

so that (5.10) also reads

$\iiint_{\Omega_{t} \times \mathcal{Y}}\left\{\sigma: B(y): \frac{\partial \sigma}{\partial \tau}+\varphi(\sigma, y)+\varphi^{*}\left(\frac{\partial}{\partial \tau}(\varepsilon-B(y): \sigma), y\right)-\sigma: \frac{\partial \varepsilon}{\partial \tau}\right\} \mathrm{d} x \mathrm{~d} y \mathrm{~d} \tau \leqslant 0$

for any $t \in] 0, T]$. By the argument at the end of $\S 2$ this inequality is equivalent to the inclusion (5.4). Thus, problem 5.1 is a weak formulation of an initial- and boundary-value problem for the system (3.20) and (5.4).

\subsection{Derivation of the two-scale problem}

Let us first denote the ratio between the two length-scales by $\eta$, so that $y=$ $x / \eta\left(y \in \mathbb{R}^{3}\right)$. However, in order to enforce the $Y$-periodicity with respect to the variable $y$ we shall let $y$ range through the three-dimensional unit torus, $\mathcal{Y}$. We thus replace the above relation by the condition

$$
y=\frac{x}{\eta} \bmod Y
$$

that is, $y \in \mathcal{Y}$ and for $i=1, \ldots, 3$ there exists $k_{i} \in \mathbb{Z}$ such that $x_{i}=k_{i} \eta+y_{i}$. The constitutive relation (5.4) thus also reads

$$
\frac{\partial \varepsilon}{\partial t}(x, t)-B\left(\frac{x}{\eta}\right): \frac{\partial \sigma}{\partial t}(x, t) \in \partial \varphi\left(\sigma(x, t), \frac{x}{\eta}\right) \quad \text { for a.e. }(x, t) \in \Omega_{T} \text { for all } \eta>0 .
$$

We couple this inclusion with the equation of continuum dynamics

$$
\rho(x) \frac{\partial^{2} \boldsymbol{u}}{\partial t^{2}}(x, t)-\nabla \cdot \sigma(x, t)=\boldsymbol{f}(x, t) \quad \text { for a.e. }(x, t) \in \Omega_{T} \text { for all } \eta>0,
$$

and formulate an initial- and boundary-value problem analogous to problem 3.1 that we then label problem $3.1_{\eta}$. By theorem 4.1, under suitable hypotheses for any $\eta>0$, this problem has a solution $\left(\boldsymbol{u}_{\eta}, \sigma_{\eta}\right)$, and by (4.15) we may assume that

$$
\begin{aligned}
& \left\|\boldsymbol{u}_{\eta}\right\|_{W^{1, \infty}\left(0, T ; L^{2}(\Omega)^{3}\right) \cap L^{q}(0, T ; V)},\left\|\sigma_{\eta}\right\|_{L^{\infty}\left(0, T ; L^{2}(\Omega)^{9}\right)},\left\|\sigma_{\eta(\mathrm{d})}\right\|_{L^{p}\left(\Omega_{T}\right)^{9}} \\
& \left.\left\|\frac{\partial}{\partial \tau}\left(\varepsilon_{\eta}-B\left(\frac{x}{\eta}\right): \sigma_{\eta}\right)\right\|_{L^{q}\left(\Omega_{T}\right)^{9}} \leqslant \text { const. (independent of } \eta\right) .
\end{aligned}
$$




\subsection{Two-scale convergence}

In view of studying the asymptotic behaviour of $\left(\boldsymbol{u}_{\eta}, \sigma_{\eta}\right)$ as $\eta$ vanishes, we briefly review the definition of two-scale convergence along the lines of the fundamental works $[4,80]$.

For any $r \in[1,+\infty]$ we say that a bounded sequence $\left\{u_{\eta}\right\}$ of $L^{r}(\Omega)$ weakly (weakly-* if $r=\infty$ ) two-scale converges to $u \in L^{r}(\Omega \times \mathcal{Y})$, and write $u_{\eta} \overrightarrow{2} u$ $\left(u_{\eta} \frac{*}{2} u\right.$ if $\left.r=\infty\right)$ whenever

$$
\int_{\Omega} u_{\eta}(x) v\left(x, \frac{x}{\eta}\right) \mathrm{d} x \rightarrow \iint_{\Omega \times \mathcal{Y}} u(x, y) v(x, y) \mathrm{d} x \mathrm{~d} y \quad \text { for all } v \in \mathcal{D}(\Omega \times \mathcal{Y}) \text {. }
$$

This is extended to time-dependent functions as follows. Let $r, s \in] 1,+\infty]$, let $\left\{u_{\eta}\right\}$ be a bounded sequence of $L^{\mathrm{s}}\left(0, T ; L^{r}(\Omega)\right)$ and let $u \in L^{\mathrm{s}}\left(0, T ; L^{r}(\Omega \times \mathcal{Y})\right.$ ). (It is known that for $r=\infty$ difficulties arise in defining these spaces, for $L^{\infty}(\Omega)$ and $L^{\infty}(\Omega \times \mathcal{Y})$ are not separable. This drawback may be removed by assuming that in this case these vector-valued functions are weakly-* measurable.) We say that $u_{\eta} \underset{2}{\longrightarrow} u\left(u_{\eta} \frac{*}{2} u\right.$ if either $r=\infty$ or $\left.s=\infty\right)$ whenever

$$
\begin{array}{r}
\iint_{\Omega_{T}} u_{\eta}(x, t) v\left(x, \frac{x}{\eta}, t\right) \mathrm{d} x \mathrm{~d} t \rightarrow \iiint_{\Omega_{T} \times \mathcal{Y}} u(x, y, t) v(x, y, t) \mathrm{d} x \mathrm{~d} y \mathrm{~d} t \\
\text { for all } v \in \mathcal{D}\left(\Omega_{T} \times \mathcal{Y}\right) .
\end{array}
$$

(A different formulation of two-scale convergence, that we do not use in this work, assumes the occurrence of a fine-scale in time, too; see, for example, [77].) The extension to either vector-valued or tensor-valued functions is fairly obvious. The next two lemmata respectively state the relative weak two-scale compactness of bounded subsets of $L^{p}$, and extend a fundamental property of $[4,80]$ about the two-scale convergence of derivatives. We remind the reader that we have assumed that $\Omega$ is a (bounded) domain of Lipschitz class.

Lemma 5.2 (Allaire [4]; Nguetseng [80]). Let $1<p \leqslant+\infty$. Then, for any bounded sequence $\left\{u_{\eta}\right\}$ of $L^{p}(\Omega)$, there exists $u \in L^{p}(\Omega \times \mathcal{Y})$ such that, possibly extracting a subsequence,

$$
u_{\eta} \underset{2}{\rightarrow} u \quad \text { in } L^{p}(\Omega \times \mathcal{Y}) \quad\left(u_{\eta} \frac{*}{2} u \quad \text { if } p=\infty\right) .
$$

LEMmA 5.3 (Visintin $[101,104])$. Let $q \in] 1,+\infty\left[\right.$ and a sequence $\left\{\boldsymbol{u}_{\eta}\right\}$ of $L^{q}(\Omega)^{3}$ be such that $\boldsymbol{u}_{\eta} \rightarrow \boldsymbol{u}$ in $W^{1, q}(\Omega)^{3}$. Then there exists $\boldsymbol{u}_{(1)} \in L^{q}\left(\Omega ; W^{1, q}(\mathcal{Y})^{3}\right)$ such that $\int_{\mathcal{Y}} \boldsymbol{u}_{(1)}(\cdot, y) \mathrm{d} y=\mathbf{0}$ a.e. in $\Omega$, and, as $\eta \rightarrow 0$ along a suitable subsequence,

$$
\nabla^{\mathrm{s}} \boldsymbol{u}_{\eta} \underset{2}{\rightarrow} \nabla^{\mathrm{s}} \boldsymbol{u}+\nabla_{y}^{\mathrm{s}} \boldsymbol{u}_{(1)} \quad \text { in } L^{q}(\Omega \times \mathcal{Y})^{9} .
$$

Lemma 5.4 (Allaire [4]; Nguetseng [80]). Let $p \in 1,+\infty$ and let a bounded sequence $\left\{w_{\eta}\right\}$ of $L^{p}(\Omega)_{\mathrm{s}}^{9}$ be such that $\left\{\eta \nabla \cdot w_{\eta}\right\}$ is bounded in $L^{p}(\Omega)^{3}$. Then there exists $w \in L^{p}(\Omega \times \mathcal{Y})_{\mathrm{s}}^{9}$ such that $\nabla_{y} \cdot w \in L^{p}(\Omega \times \mathcal{Y})^{3}$, and, as $\eta \rightarrow 0$ along a suitable subsequence,

$$
w_{\eta} \underset{2}{\rightarrow} w \in L^{p}(\Omega \times \mathcal{Y})_{\mathrm{s}}^{9}, \quad \eta \nabla \cdot w_{\eta} \underset{2}{\rightarrow} \nabla_{y} \cdot w \in L^{p}(\Omega \times \mathcal{Y})^{3} .
$$


These results may trivially be extended to time-dependent functions.

We are now able to show the two-scale convergence of the solutions of problem $3.1_{\eta}$ to a solution of problem 5.1 as $\eta$ vanishes (along a subsequence).

THEOREM 5.5. Assume that the hypotheses (5.1)-(5.3), (5.6), (5.7) are satisfied. For any $\eta$ let $\left(\boldsymbol{u}_{\eta}, \sigma_{\eta}\right)$ be a solution of problem $3.1_{\eta}$ that satisfies the estimates (5.16). Then there exist $\boldsymbol{u}, \varepsilon, \sigma$ such that, as $\eta \rightarrow 0$ along a suitable subsequence,

$$
\begin{gathered}
\boldsymbol{u}_{\eta} \stackrel{*}{\rightarrow} \boldsymbol{u} \quad \text { in } W^{1, \infty}\left(0, T ; L^{2}(\Omega)^{3}\right) \cap L^{q}(0, T ; V), \\
\varepsilon_{\eta} \underset{2}{\rightarrow} \quad \text { in } L^{q}\left(\Omega_{T} \times \mathcal{Y}\right)^{9}, \\
\sigma_{\eta} \frac{*}{2} \sigma \quad \text { in } L^{\infty}\left(0, T ; L^{2}(\Omega \times \mathcal{Y})^{9}\right), \\
\sigma_{\eta(\mathrm{d})} \underset{2}{\rightarrow} \sigma_{(\mathrm{d})} \quad \text { in } L^{p}\left(\Omega_{T} \times \mathcal{Y}\right)^{9} \\
\frac{\partial}{\partial \tau}\left(\varepsilon_{\eta}-B\left(\frac{x}{\eta}\right): \sigma_{\eta}\right) \underset{2}{\rightarrow} \frac{\partial}{\partial \tau}(\varepsilon-B(y): \sigma) \quad \text { in } L^{q}\left(\Omega_{T} \times \mathcal{Y}\right)^{9} .
\end{gathered}
$$

This implies that $(\boldsymbol{u}, \varepsilon, \sigma)$ is a solution of problem 5.1 .

Proof. By lemma 5.2 the convergences (5.22)-(5.26) follow from the uniform estimates (5.16). The properties (5.8) are thus established. By lemma 5.3 the first of the estimates (5.16) also yields (5.9).

As the semicontinuity properties of weak single-scale convergence are extended to weak two-scale convergence (see [105, proposition 1.3(iii)]), we have

$$
\begin{gathered}
\liminf _{\eta \rightarrow 0} \int_{\Omega} \sigma_{\eta}(x, t): B\left(\frac{x}{\eta}\right): \sigma_{\eta}(x, t) \mathrm{d} x \geqslant \iint_{\Omega \times \mathcal{Y}} \sigma(x, y, t): B(y): \sigma(x, y, t) \mathrm{d} x \mathrm{~d} y, \\
\liminf _{\eta \rightarrow 0} \iint_{\Omega_{t}}\left[\varphi\left(\sigma_{\eta}, \frac{x}{\eta}\right)+\varphi^{*}\left(\frac{\partial}{\partial \tau}\left(\varepsilon_{\eta}-B\left(\frac{x}{\eta}\right): \sigma_{\eta}\right), \frac{x}{\eta}\right)\right] \mathrm{d} x \mathrm{~d} \tau \\
\left.\left.\geqslant \iiint_{\Omega_{t} \times \mathcal{Y}}\left[\varphi(\sigma, y)+\varphi^{*}\left(\frac{\partial}{\partial \tau}(\varepsilon-B(y): \sigma), y\right)\right] \mathrm{d} x \mathrm{~d} y \mathrm{~d} \tau \text { for a.e. } t \in\right] 0, T\right] .
\end{gathered}
$$

By writing (3.18) and (3.19) for any $\eta$ and passing to the inferior limit as $\eta \rightarrow 0$, we then get (5.10) and (5.11). Writing (3.20) for any $\eta$ and comparing the terms of this equation, we see that $\nabla \cdot \sigma_{\eta}$ is uniformly bounded in $H^{-1}\left(0, T ; L^{2}(\Omega)^{3}\right)$; lemma 5.4 then yields (5.12).

THEOREM 5.6 (regularity and uniqueness). Let (5.1)-(5.3), (5.6) be satisfied and

$$
\left.\begin{array}{c}
\boldsymbol{u}^{0}, \boldsymbol{v}^{0} \in L^{2}(\Omega)^{3} \cap V, \quad \boldsymbol{f} \in W^{1,1}\left(0, T ; L^{2}(\Omega)^{3}\right), \\
\partial \varphi\left(\sigma^{0}, y\right) \cap L^{2}(\Omega \times \mathcal{Y})_{\mathrm{s}}^{9} \neq \emptyset, \quad \boldsymbol{f}(0)+\nabla^{*} \cdot \hat{\sigma}^{0} \in L^{2}(\Omega)^{3} .
\end{array}\right\}
$$

Then problem 5.1 has one and only one solution $(\boldsymbol{u}, \varepsilon, \sigma)$ such that

$$
\left.\begin{array}{c}
\boldsymbol{u} \in W^{2, \infty}\left(0, T ; L^{2}(\Omega)^{3}\right) \cap W^{1, \infty}(0, T ; V), \\
W^{1, \infty}\left(0, T ; L^{2}(\Omega \times \mathcal{Y})_{\mathrm{s}}^{9}\right), \quad \nabla \cdot \hat{\sigma} \in L^{2}\left(\Omega_{T}\right)^{3} .
\end{array}\right\}
$$

(Equation (3.20) is thus satisfied a.e. in $\Omega_{T}$; namely, $(\boldsymbol{u}, \varepsilon, \sigma)$ is a strong solution.) 
Proof. By theorem 4.3 for any $\eta>0$, problem $3.1_{\eta}$ has a solution $\left(\boldsymbol{u}_{\eta}, \sigma_{\eta}\right)$ with the regularity (4.23) that satisfies the uniform estimates (4.27) and (4.28) (with the index $\eta$ in place of $m$ ). This yields the first two inclusions of (5.30) for the two-scale limit of these sequences. The third inclusion follows from comparing the terms of equation (3.20). The uniqueness of the solution may be checked by mimicking the argument of theorem 4.5 .

A property of continuous dependence on the data analogous to (4.29) may be proved for problem 5.1, too.

\subsection{Limit cases}

Theorem 5.5 may easily be extended to the quasi-static problem, either directly or by letting the density $\rho$ vanish in the dynamical problem.

In a univariate system $\nabla^{\mathrm{s}}$ and $\nabla$. are both replaced by the single derivative $D_{x}$ and, similarly, $\nabla_{y}^{\mathrm{s}}$ and $\nabla_{y}$. are replaced by $D_{y}$. Equation (5.12) then just reads $D_{y} \sigma=0$ in $\mathcal{D}^{\prime}\left(\Omega_{T} \times \mathcal{Y}\right)$, so that $\sigma$ is independent of $y$ (i.e. $\hat{\sigma}=\sigma$ ), at variance with the multi-axial problem. On the other hand, in general, $\varepsilon$ should be expected to depend on $y$, since no uniform estimate on $D \varepsilon_{\eta}$ seems available. Finally, note that in uniaxial systems the quasi-static equation is reduced to $-D \sigma=\boldsymbol{f}$, which can be integrated directly.

\subsection{Elastoplasticity without strain-hardening}

As we saw, the classical Prandtl-Reuss model of elastoplasticity without strainhardening corresponds to $\varphi=I_{K}, K$ being a closed convex subset of $D_{\mathrm{s}}^{9}$ with $0 \in K$. Here we illustrate the associated two-scale model.

First we extend the notion of two-scale convergence to measures along the lines of [6]. Let us denote by $C_{0}^{0}(\bar{\Omega} \times \mathcal{Y})$ the space of continuous functions $\bar{\Omega} \times \mathcal{Y} \rightarrow \mathbb{R}$ that vanish on $(\partial \Omega) \times \mathcal{Y}$ (namely the boundary of $\Omega \times \mathcal{Y}$, for $\mathcal{Y}$ has no boundary); this is a Banach space equipped with the maximum norm. We say that a bounded sequence of Radon measures $\left\{\mu_{\eta}\right\} \subset C_{0}^{0}(\bar{\Omega})^{\prime}$ weakly-* two-scale converges to a measure $\mu \in C_{0}^{0}(\bar{\Omega} \times \mathcal{Y})^{\prime}$, and write $\mu_{\eta} \frac{*}{2} \mu$ in $C_{0}^{0}(\bar{\Omega} \times \mathcal{Y})^{\prime}$, if

$$
\int_{\Omega} v\left(x, \frac{x}{\eta}\right) \mu_{\eta}(\mathrm{d} x) \rightarrow \iint_{\Omega \times \mathcal{Y}} v(x, y) \mu(\mathrm{d} x \mathrm{~d} y) \quad \text { for all } v \in C_{0}^{0}(\bar{\Omega} \times \mathcal{Y}) \text {. }
$$

This definition might easily be extended to measures of $C_{0}^{0}\left(\overline{\Omega_{T}}\right)^{\prime}$, assuming either a single fine-scale (space) variable, $y$, or two fine-scale (space and time) variables, $y$ and $\tau$. However, consistently with the above developments, here we just deal with the former case. We thus say that $\left\{\mu_{\eta}\right\} \subset C_{0}^{0}\left(\overline{\Omega_{T}}\right)^{\prime}$ weakly-* two-scale converges to $\mu \in C_{0}^{0}\left(\overline{\Omega_{T}} \times \mathcal{Y}\right)^{\prime}$ if

$$
\begin{array}{r}
\iint_{\Omega_{T}} v\left(x, \frac{x}{\eta}, t\right) \mu_{\eta}(\mathrm{d} x \mathrm{~d} t) \rightarrow \iiint_{\Omega_{T} \times \mathcal{Y}} v(x, y, t) \mu(\mathrm{d} x \mathrm{~d} y \mathrm{~d} t) \\
\quad \text { for all } v \in C_{0}^{0}\left(\overline{\Omega_{T}} \times \mathcal{Y}\right) .
\end{array}
$$

The further extension to either vector-valued or tensor-valued measures is obvious. The next two statements may be compared with lemmata 5.2 and 5.3. 
Lemma 5.7 (Amar [6]). For any bounded sequence $\left\{\mu_{\eta}\right\}$ of $C_{0}^{0}\left(\overline{\Omega_{T}}\right)^{\prime}$ there exists $\mu \in C_{0}^{0}\left(\overline{\Omega_{T}} \times \mathcal{Y}\right)^{\prime}$ such that, possibly extracting a subsequence,

$$
\mu_{\eta} \underset{2}{*} \mu \quad \text { in } C_{0}^{0}\left(\overline{\Omega_{T}} \times \mathcal{Y}\right)^{\prime} .
$$

This statement is trivially extended to either vector-valued or tensor-valued measures.

Lemma 5.8 (Amar [6]). Let a sequence $\left\{\boldsymbol{\mu}_{\eta}\right\}$ of $X\left(\Omega_{T}\right)$ (see (3.27)) be bounded in this space, and such that $\boldsymbol{\mu}_{\eta} * \boldsymbol{\mu}$ in $\left(C_{0}^{0}\left(\overline{\Omega_{T}}\right)^{3}\right)^{\prime}$. Then there exists $\boldsymbol{\mu}_{(1)} \in$ $\left(C_{0}^{0}\left(\overline{\Omega_{T}} \times \mathcal{Y}\right)^{3}\right)^{\prime}$ such that

$$
\int_{\mathcal{Y}} \boldsymbol{\mu}_{(1)}(\cdot, y)=\mathbf{0} \quad \text { in }\left(C_{0}^{0}\left(\overline{\Omega_{T}}\right)^{3}\right)^{\prime}, \quad \nabla_{y}^{\mathrm{s}} \boldsymbol{\mu}_{(1)} \in\left(C_{0}^{0}\left(\overline{\Omega_{T}} \times \mathcal{Y}\right)^{9}\right)^{\prime},
$$

and, as $\eta \rightarrow 0$ along a suitable subsequence,

$$
\nabla^{\mathrm{s}} \boldsymbol{\mu}_{\eta} \underset{2}{\rightarrow} \nabla^{\mathrm{s}} \boldsymbol{\mu}+\nabla_{y}^{\mathrm{s}} \boldsymbol{\mu}_{(1)} \quad \text { in }\left(C_{0}^{0}\left(\overline{\Omega_{T}} \times \mathcal{Y}\right)^{9}\right)^{\prime} .
$$

Next we assume that the Dirichlet part of the boundary, $\Gamma_{0}$, is empty, and reformulate problem 5.1 with the following amendments. The data $\boldsymbol{u}^{0}$ and $\boldsymbol{u}_{(1)}^{0}$ of (5.6) are here replaced by two measures $\boldsymbol{\mu}^{0}$ and $\boldsymbol{\mu}_{(1)}^{0}$ such that

$$
\begin{aligned}
\boldsymbol{\mu}^{0} & \in\left(C_{0}^{0}\left(\overline{\Omega_{T}}\right)^{3}\right)^{\prime}, & \nabla^{\mathrm{s}} \boldsymbol{\mu}^{0} & \in\left(C_{0}^{0}\left(\overline{\Omega_{T}}\right)^{9}\right)^{\prime}, \\
\boldsymbol{\mu}_{(1)}^{0} & \in\left(C_{0}^{0}\left(\overline{\Omega_{T}} \times \mathcal{Y}\right)^{3}\right)^{\prime}, & \nabla_{y}^{\mathrm{s}} \boldsymbol{\mu}_{(1)}^{0} & \in\left(C_{0}^{0}\left(\overline{\Omega_{T}} \times \mathcal{Y}\right)^{9}\right)^{\prime} .
\end{aligned}
$$

The final inclusion of (5.8) and the hypothesis (5.9) are then replaced by

$$
\begin{gathered}
\varepsilon, \frac{\partial}{\partial t}(\varepsilon-B(y): \sigma) \in\left(C_{0}^{0}\left(\overline{\Omega_{T}} \times \mathcal{Y}\right)^{9}\right)^{\prime}, \\
\exists \boldsymbol{\mu}_{(1)} \in\left(C_{0}^{0}\left(\overline{\Omega_{T}} \times \mathcal{Y}\right)^{3}\right)^{\prime}: \varepsilon=\nabla^{\mathrm{s}} \boldsymbol{\mu}+\nabla_{y} \boldsymbol{\mu}_{(1)} \quad \text { in }\left(C_{0}^{0}\left(\overline{\Omega_{T}} \times \mathcal{Y}\right)^{9}\right)^{\prime} .
\end{gathered}
$$

Setting $\xi=\varepsilon-B(y): \sigma$, the term of (5.10) containing $\varphi^{*}$ is then replaced by

$$
\iiint_{\Omega_{t} \times \mathcal{Y}} \varphi^{*}\left(D_{t} \xi, y\right)=\iiint_{\Omega_{t} \times \mathcal{Y}} \varphi^{*}\left(\frac{D_{t} \xi}{\left|D_{t} \xi\right|}, y\right)\left|D_{t} \xi(\mathrm{d} x \mathrm{~d} y \mathrm{~d} t)\right| .
$$

The existence and regularity results (theorems 5.5 and 5.6) then are extended to this setting with obvious amendments, whereas for the well-posedness one faces the difficulties that we mentioned at the end of $\S 4$.

Remark 5.9. As $B$ and $\varphi$ explicitly depend on $y$ but not on $x$, the relation (5.4) may be interpreted as the constitutive behaviour of a macroscopically homogeneous and mesoscopically non-homogeneous viscoelastic material. The developments of this paper may easily be extended to a material that is both macroscopically and mesoscopically non-homogeneous, by allowing $B$ and $\varphi$ to depend explicitly on both $x$ and $y$.

\section{Homogenization of the constitutive law}

In the remainder of this paper we confine ourselves to the (nonlinear) Maxwell model for $p=q=2$, as some technical difficulties seem to arise in the more 
general setting. In this section we derive a purely coarse-scale constitutive relation by eliminating any dependence on the fine-scale variable $y \in \mathcal{Y}$ in the two-scale system

$$
\left.\begin{array}{rl}
\frac{\partial \varepsilon}{\partial t}(x, y, t)-B(y): \frac{\partial \sigma}{\partial t}(x, y, t) \in \partial \varphi(\sigma(x, y, t), y) & \text { for a.e. }(x, y, t) \in \Omega_{T} \times \mathcal{Y}, \\
\varepsilon(x, y, 0)=B(y): \sigma(x, y, 0) & \text { for a.e. }(x, y) \in \Omega \times \mathcal{Y},
\end{array}\right\}
$$

in view of applying a similar scale reduction (so-called upscaling) to the whole twoscale problem 5.1 in the next section. By and large this procedure may be compared with that of [103, $\S \S 4$ and 5].

Henceforth, we assume that (5.2) and (5.3) are satisfied with $p=q=2$. (We do so because in the weak formulation the deviatoric part of the stress tensor $\sigma$ is only known to belong to $L^{p}\left(\Omega_{T} \times \mathcal{Y}\right)_{\mathrm{s}}^{9}$.) As in $(6.1)$, the coarse-scale variable $x$ just plays the role of a parameter, for the sake of simplicity here we omit it, and just study a cell problem. We also set

$$
r=\frac{\partial \varepsilon}{\partial t}, \quad s=\frac{\partial \sigma}{\partial t}
$$

and first deal with the stationary inclusion

$$
r(y)-B(y): s(y) \in \partial \varphi(\sigma(y), y) \text { for a.e. } y \in \mathcal{Y},
$$

assuming that $r$ and $s$ are independent of $\varepsilon$ and $\sigma$. We shall prescribe the relations (6.2) only afterwards.

We anticipate a result that we shall use ahead in this section.

LEMma 6.1. Let $B_{1}, B_{2}$ be real vector spaces, $f$ be a mapping $\left.\left.B_{1} \times B_{2} \rightarrow\right]-\infty,+\infty\right]$, and define the infimal value function

$$
g: B_{1} \rightarrow[-\infty,+\infty]: \xi_{1} \mapsto \inf \left\{f\left(\xi_{1}, \xi_{2}\right): \xi_{2} \in B_{2}\right\}
$$

Then

(i) if $f$ is convex then $g$ is also convex,

(ii) if $B_{1}, B_{2}$ are real normed spaces and $f$ is coercive, that is, $\left\{\left(\xi_{1}, \xi_{2}\right) \in B_{1} \times B_{2}\right.$ : $\left.f\left(\xi_{1}, \xi_{2}\right) \leqslant M\right\}$ is bounded for any $M>0$, then $g$ is also coercive,

(iii) let $B_{1}, B_{2}$ be real Banach spaces, $B_{2}$ be reflexive and $f$ be coercive with respect to $\xi_{2}$ locally uniformly with respect to $\xi_{1}$, in the sense that

for all bounded $S \subset B_{1}$ and for all $M>0$,

$$
\left\{\xi_{2} \in B_{2}: f\left(\xi_{1}, \xi_{2}\right) \leqslant M \text { for all } \xi_{1} \in S\right\} \text { is bounded; }
$$

under these further assumptions, if $f$ is lower semicontinuous, then $g$ is also lower semicontinuous. 
Proof.

(i) For any $\xi_{1}^{\prime}, \xi_{1}^{\prime \prime} \in B_{1}$, any $\xi_{2}^{\prime}, \xi_{2}^{\prime \prime} \in B_{2}$ and any $\left.\lambda \in\right] 0,1[$, by the convexity of $f$ we have

$$
\begin{aligned}
g\left(\lambda \xi_{1}^{\prime}+(1-\lambda) \xi_{1}^{\prime \prime}\right) & \leqslant f\left(\lambda \xi_{1}^{\prime}+(1-\lambda) \xi_{1}^{\prime \prime}, \lambda \xi_{2}^{\prime}+(1-\lambda) \xi_{2}^{\prime \prime}\right) \\
& =f\left(\lambda\left(\xi_{1}^{\prime}, \xi_{2}^{\prime}\right)+(1-\lambda)\left(\xi_{1}^{\prime \prime}, \xi_{2}^{\prime \prime}\right)\right) \\
& \leqslant \lambda f\left(\xi_{1}^{\prime}, \xi_{2}^{\prime}\right)+(1-\lambda) f\left(\xi_{1}^{\prime \prime}, \xi_{2}^{\prime \prime}\right) .
\end{aligned}
$$

By taking the infimum with respect to $\xi_{2}^{\prime}, \xi_{2}^{\prime \prime}$, we then get $g\left(\lambda \xi_{1}^{\prime}+(1-\lambda) \xi_{1}^{\prime \prime}\right) \leqslant$ $\lambda g\left(\xi_{1}^{\prime}\right)+(1-\lambda) g\left(\xi_{1}^{\prime \prime}\right)$.

(ii) The statement concerning the coerciveness is straightforward.

(iii) Let us fix any sequence $\left\{\xi_{1 n}\right\}$ in $B_{1}$ that weakly converges to some $\xi_{1} \in B_{1}$; thus, $\left\{\xi_{1 n}\right\}$ is bounded. If $L:=\liminf _{n \rightarrow \infty} g\left(\xi_{1 n}\right)=+\infty$, then trivially $g\left(\xi_{1}\right) \leqslant L$. If, instead, $L<+\infty$, then by definition of $g$ there exists a sequence $\left\{\xi_{2 n}\right\}$ in $B_{2}$ such that (at least for sufficiently large $n$ )

$$
f\left(\xi_{1 n}, \xi_{2 n}\right) \leqslant \begin{cases}g\left(\xi_{1 n}\right)+\frac{1}{n} & \text { for all } n \in \mathbb{N} \text { if } L>-\infty \\ -n & \text { for all } n \in \mathbb{N} \text { if } L=-\infty\end{cases}
$$

By (6.5) this sequence is confined to a bounded subset of the reflexive space $B_{2}$. Hence, there exists $\xi_{2} \in B_{2}$ such that, as $n$ diverges along a further subsequence, $\xi_{2 n} \rightarrow \xi_{2}$ weakly in $B_{2}$. Passing to the limit in (6.6) on this subsequence, by the lower semicontinuity of $f$ we then get

$$
g\left(\xi_{1}\right) \leqslant f\left(\xi_{1}, \xi_{2}\right) \leqslant \liminf _{n \rightarrow \infty} f\left(\xi_{1 n}, \xi_{2 n}\right) \leqslant \liminf _{n \rightarrow \infty} g\left(\xi_{1 n}\right) .
$$

Next we address the homogenization of the inclusion (6.3). We split our argument into several steps, for the reader's convenience.

STEP 1. Using the decomposition (5.5), let us first define the Hilbert spaces

$$
\begin{aligned}
W & :=\left\{\eta \in L^{2}(\mathcal{Y})_{\mathrm{s}}^{9}: \hat{\eta}=0, \nabla \cdot \eta=\mathbf{0} \text { in } \mathcal{D}^{\prime}(\mathcal{Y})^{3}\right\}, \\
Z & :=\left\{\zeta \in L^{2}(\mathcal{Y})_{\mathrm{s}}^{9}: \hat{\zeta}=0, \zeta=\nabla^{\mathrm{s}} \boldsymbol{v} \text { a.e. in } \mathcal{Y} \text { for some } \boldsymbol{v} \in H^{1}(\mathcal{Y})^{3}\right\},
\end{aligned}
$$

and note that

$$
\left.\begin{array}{cc}
\int_{\mathcal{Y}} \zeta(y): \eta(y) \mathrm{d} y=0 & \text { for all } \zeta \in Z, \text { for all } \eta \in W, \\
\int_{\mathcal{Y}} \hat{\zeta}: \tilde{\eta}(y) \mathrm{d} y=0 & \text { for all } \zeta, \eta \in L^{2}(\mathcal{Y})^{9} .
\end{array}\right\}
$$

STEP 2. The properties (2.12) and (2.14) respectively yield

$$
\begin{gathered}
\varphi(\sigma, y)+\varphi^{*}(r-B(y): s, y) \geqslant \sigma:(r-B(y): s) \quad \forall(\sigma, r, s) \in\left(\mathbb{R}_{\mathrm{s}}^{9}\right)^{3} \text { for a.e. } y \in \mathcal{Y} \\
(6.10) \\
(6.3) \Longleftrightarrow \quad \begin{array}{l}
\varphi(\sigma(y), y)+\varphi^{*}(r(y)-B(y): s(y), y) \\
\leqslant \sigma(y):(r(y)-B(y): s(y)) \quad \text { for a.e. } y \in \mathcal{Y} .
\end{array}
\end{gathered}
$$


The inequality (6.10) also reads

$$
\begin{aligned}
\varphi(\bar{\sigma}+\tilde{\sigma}, y)+\varphi^{*}(\bar{r}+\tilde{r}-B(y): & (\bar{s}+\tilde{s}), y)+(\bar{\sigma}+\tilde{\sigma}): B(y):(\bar{s}+\tilde{s}) \geqslant(\bar{\sigma}+\tilde{\sigma}):(\bar{r}+\tilde{r}) \\
& \text { for all }(\bar{\sigma}, \bar{r}, \bar{s}, \tilde{\sigma}, \tilde{r}, \tilde{s}) \in\left(\mathbb{R}_{\mathrm{s}}^{9}\right)^{6} \text { for a.e. } y \in \mathcal{Y} .
\end{aligned}
$$

STEP 3. Let us now set

$$
\begin{array}{r}
U(\sigma, r, s):=\int_{\mathcal{Y}}\left\{\varphi(\sigma(y), y)+\varphi^{*}(r(y)-B(y): s(y), y)+\sigma(y): B(y): s(y)\right\} \mathrm{d} y \\
\text { for all }(\sigma, r, s) \in\left(\mathbb{R}_{\mathrm{s}}^{9}+W\right) \times\left(\mathbb{R}_{\mathrm{s}}^{9}+Z\right) \times\left(\mathbb{R}_{\mathrm{s}}^{9}+W\right) ; \quad(6.1
\end{array}
$$

by (5.3) this integral either converges or diverges to $+\infty$. By the orthogonality properties (6.9) the inequality (6.12) yields

$$
\begin{aligned}
U(\sigma, r, s) & \geqslant \int_{\mathcal{Y}}(\hat{\sigma}+\tilde{\sigma}):(\hat{r}+\tilde{r}) \mathrm{d} y \\
& =\hat{\sigma}: \hat{r} \quad \text { for all }(\sigma, r, s) \in\left(\mathbb{R}_{\mathrm{s}}^{9}+W\right) \times\left(\mathbb{R}_{\mathrm{s}}^{9}+Z\right) \times\left(\mathbb{R}_{\mathrm{s}}^{9}+W\right) .
\end{aligned}
$$

STEP 4. The statement (6.11) also reads

$$
\begin{aligned}
(6.3) \Longleftrightarrow \varphi(\sigma(y), y)+\varphi^{*}(r(y)-B(y): s(y), y)+\sigma(y): B(y): s(y) & \\
& \leqslant[\hat{\sigma}+\tilde{\sigma}(y)]:[\hat{r}+\tilde{r}(y)] \text { for a.e. } y \in \mathcal{Y} .
\end{aligned}
$$

Moreover, by (6.10) this inequality is also equivalent to the corresponding equality. Integrating it over $\mathcal{Y}$ and using (6.9), we then get

$$
\Longleftrightarrow U(\sigma, r, s) \leqslant \hat{\sigma}: \hat{r} \Longleftrightarrow U(\sigma, r, s)=\hat{\sigma}: \hat{r} .
$$

(Note that the first implication ' $\Leftarrow$ ' is also based on the inequality (6.12).)

STEP 5. Next we come to the Cauchy problem (6.1): we shall characterize it just in terms of the average fields $\hat{\sigma}$ and $\hat{\varepsilon}$, independently of the fluctuating components $\tilde{\sigma}$ and $\tilde{\varepsilon}$. Here we reintroduce the time-dependence (in the remainder of this section we shall denote the distributional partial differentiation in time by the index $t$, in order to simplify the display of formulae), and first set

$$
\begin{aligned}
\Sigma:=\left\{(\sigma, \varepsilon) \in\left(L^{2}\left(\mathcal{Y}_{T}\right)_{\mathrm{s}}^{9}\right)^{2}:(\tilde{\sigma}, \tilde{\varepsilon}) \in L^{2}(0, T ; W \times Z),\right. & \\
& \left.\sqrt{T-t}[\varepsilon-B(y): \sigma]_{t} \in L^{2}\left(\mathcal{Y}_{T}\right)^{9},[\varepsilon-B(y): \sigma](\cdot, 0)=0 \text { a.e. in } \mathcal{Y}\right\},
\end{aligned}
$$

which is a Hilbert space equipped with the graph norm

$$
\|(\sigma, \varepsilon)\|_{1}:=\|\sigma\|_{L^{2}\left(\mathcal{Y}_{T}\right)^{9}}+\|\varepsilon\|_{L^{2}\left(\mathcal{Y}_{T}\right)^{9}}+\left\|\sqrt{T-t}[\varepsilon-B(y): \sigma]_{t}\right\|_{L^{2}\left(\mathcal{Y}_{T}\right)^{9}} .
$$

We claim that this norm is equivalent to the reduced norm

$$
\|(\sigma, \varepsilon)\|_{\Sigma}:=\|\sigma\|_{L^{2}\left(\mathcal{Y}_{T}\right)^{9}}+\left\|\sqrt{T-t}[\varepsilon-B(y): \sigma]_{t}\right\|_{L^{2}\left(\mathcal{Y}_{T}\right)^{9}}
$$

Indeed, for any $v \in H^{1}(0, T)$ and any $\left.t \in\right] 0, T[$, setting

$$
J(v, t):=\left(\int_{0}^{t}(T-\tau)\left|v^{\prime}(\tau)\right|^{2} \mathrm{~d} \tau\right)^{1 / 2},
$$


by the Cauchy-Schwarz inequality we have

$$
|v(t)-v(0)|=\left|\int_{0}^{t} v^{\prime}(\tau) \mathrm{d} \tau\right| \leqslant\left(\int_{0}^{t} \frac{\mathrm{d} \tau}{T-\tau}\right)^{1 / 2} J(v, t) \leqslant\left(\log \frac{T}{T-t}\right)^{1 / 2} J(v, T) .
$$

Setting $C=\left(\int_{0}^{T} \log (T /(T-t)) \mathrm{d} t\right)^{1 / 2}$, we then get

$$
\|v-v(\cdot, 0)\|_{L^{2}(0, T)} \leqslant C J(v, T)=C\left\|\sqrt{T-t} v^{\prime}(t)\right\|_{L^{2}(0, T)} .
$$

By applying this inequality to $v=\varepsilon-B(y): \sigma$ and recalling that $B \in L^{\infty}(\mathcal{Y} ; \mathcal{M})$, we infer that

$$
\|(\sigma, \varepsilon)\|_{\Sigma} \rightarrow+\infty \quad \Longleftrightarrow\|(\sigma, \varepsilon)\|_{1} \rightarrow+\infty
$$

these two norms are thus equivalent.

SteP 6 . Let us now fix any $\varepsilon^{0} \in \mathbb{R}_{\mathrm{s}}^{9}+Z$ and any $\sigma^{0} \in \mathbb{R}_{\mathrm{s}}^{9}+W$ and set

$$
\begin{aligned}
& \Sigma\left(\sigma^{0}, \varepsilon^{0}\right):=\left\{(\sigma, \varepsilon) \in\left(L^{2}\left(\mathcal{Y}_{T}\right)_{\mathrm{s}}^{9}\right)^{2}:(\tilde{\sigma}, \tilde{\varepsilon}) \in L^{2}(0, T ; W \times Z),\right. \\
& \sqrt{T-t}[\varepsilon-B(y): \sigma]_{t} \in L^{2}\left(\mathcal{Y}_{T}\right)^{9}, \\
& \left.[\varepsilon-B(y): \sigma](\cdot, 0)=\varepsilon^{0}-B(y): \sigma^{0} \text { a.e. in } \mathcal{Y}\right\}, \\
& \Xi\left(\sigma^{0}, \varepsilon^{0}\right):=\left\{(\sigma, \varepsilon) \in \Sigma\left(\sigma^{0}, \varepsilon^{0}\right): \sqrt{T-t} \hat{\varepsilon}_{t} \in L^{2}(0, T)^{9}\right\}, \\
& A(\sigma, \varepsilon):=\left\{\begin{array}{cc}
\iint_{\mathcal{Y}_{T}}\left\{(T-t)\left[\varphi(\sigma(y, t), y)+\varphi^{*}\left([\varepsilon-B(y): \sigma]_{t}(y, t), y\right)\right]\right. \\
\left.+\frac{1}{2} \sigma(y, t): B(y): \sigma(y, t)-\frac{1}{2} \sigma^{0}(y): B(y): \sigma^{0}(y)\right\} \mathrm{d} y \mathrm{~d} t \\
\text { for all }(\sigma, \varepsilon) \in \Sigma\left(\sigma^{0}, \varepsilon^{0}\right), \\
+\infty & \text { for all }(\sigma, \varepsilon) \in\left(L^{2}\left(\mathcal{Y}_{T}\right)_{\mathrm{s}}^{9}\right)^{2} \backslash \Sigma\left(\sigma^{0}, \varepsilon^{0}\right),
\end{array}\right. \\
& \Lambda(\bar{\sigma}, \bar{\varepsilon}):=\inf \left\{A(\bar{\sigma}+\tilde{\sigma}, \bar{\varepsilon}+\tilde{\varepsilon}):(\tilde{\sigma}, \tilde{\varepsilon}) \in L^{2}(0, T ; W \times Z)\right\} \\
& \text { for all }(\bar{\sigma}, \bar{\varepsilon}) \in\left(L^{2}(0, T)_{\mathrm{s}}^{9}\right)^{2} .
\end{aligned}
$$

Note that $\Sigma\left(\sigma^{0}, \varepsilon^{0}\right)$ is an affine space for $\Sigma\left(\sigma^{0}, \varepsilon^{0}\right)=\Sigma+\varepsilon^{0}-B: \sigma^{0}$; it may then be equipped with the topology induced by $\Sigma$.

STEP 7. The inequality (6.14) yields

$$
A(\sigma, \varepsilon) \geqslant \int_{0}^{T}(T-t) \hat{\sigma}: \hat{\varepsilon}_{t} \mathrm{~d} t \quad \text { for all }(\sigma, \varepsilon) \in \Xi\left(\sigma^{0}, \varepsilon^{0}\right) .
$$

Setting

$$
P\left(\varepsilon^{0}\right):=\left\{(\bar{\sigma}, \bar{\varepsilon}) \in\left(L^{2}(0, T)_{\mathrm{s}}^{9}\right)^{2}: \sqrt{T-t} \bar{\varepsilon}_{t} \in L^{2}(0, T)^{9}, \bar{\varepsilon}(0)=\hat{\varepsilon}^{0}\right\}
$$


we then have

$$
\Lambda(\bar{\sigma}, \bar{\varepsilon}) \geqslant \int_{0}^{T}(T-t) \bar{\sigma}: \bar{\varepsilon}_{t} \mathrm{~d} t \quad \text { for all }(\bar{\sigma}, \bar{\varepsilon}) \in P\left(\varepsilon^{0}\right) .
$$

Moreover, for any $(\sigma, \varepsilon) \in \Xi\left(\sigma^{0}, \varepsilon^{0}\right)$ by $(6.15)$ the inclusion

$$
[\varepsilon-B(y): \sigma]_{t} \in \partial \varphi(\sigma, y) \quad \text { a.e. in } \mathcal{Y}_{T}
$$

is equivalent to

$$
A(\sigma, \varepsilon)=\int_{0}^{T}(T-t) \hat{\sigma}: \hat{\varepsilon}_{t} \mathrm{~d} t
$$

By (6.23) it then follows that, for any $(\sigma, \varepsilon) \in \Xi\left(\sigma^{0}, \varepsilon^{0}\right)$,

$$
(6.25) \quad \Longrightarrow \quad \Lambda(\hat{\sigma}, \hat{\varepsilon})=\int_{0}^{T}(T-t) \hat{\sigma}: \hat{\varepsilon}_{t} \mathrm{~d} t .
$$

Step 8. We claim that, conversely, for any $\varepsilon^{0} \in \mathbb{R}_{\mathrm{s}}^{9}+Z$ and any $\sigma^{0} \in \mathbb{R}_{\mathrm{s}}^{9}+W$, if the pair $(\bar{\sigma}, \bar{\varepsilon}) \in P\left(\varepsilon^{0}\right)$ is such that

$$
\Lambda(\bar{\sigma}, \bar{\varepsilon})=\int_{0}^{T}(T-t) \bar{\sigma}: \bar{\varepsilon}_{t} \mathrm{~d} t \quad\left(\text { or, equivalently, } \Lambda(\bar{\sigma}, \bar{\varepsilon}) \leqslant \int_{0}^{T}(T-t) \bar{\sigma}: \bar{\varepsilon}_{t} \mathrm{~d} t\right)
$$

then there exists a pair $(\tilde{\sigma}, \tilde{\varepsilon}) \in L^{2}(0, T ; W \times Z)$ such that $(\sigma, \varepsilon):=(\bar{\sigma}+\tilde{\sigma}, \bar{\varepsilon}+\tilde{\varepsilon}) \in$ $\Xi\left(\sigma^{0}, \varepsilon^{0}\right)$ and $(6.25)$ is satisfied.

In view of justifying this claim, let us first note that the functional $A$ is bounded from below, convex and lower semicontinuous. Let us set

$$
\left.\left.\mathcal{A}:\left(L^{2}\left(\mathcal{Y}_{T}\right)_{\mathrm{s}}^{9}\right)^{2} \times L^{2}(0, T ; W \times Z) \rightarrow\right]-\infty,+\infty\right]:(\bar{\sigma}, \bar{\varepsilon}, \tilde{\sigma}, \tilde{\varepsilon}) \mapsto A(\bar{\sigma}+\tilde{\sigma}, \bar{\varepsilon}+\tilde{\varepsilon}) .
$$

By (5.3) (here with $p=q=2$ ) this functional is coercive in the sense of (6.5) with $\xi_{1}=(\bar{\sigma}, \bar{\varepsilon})$ and $\xi_{2}=(\tilde{\sigma}, \tilde{\varepsilon})$. By lemma 6.1 the functional $\Lambda$ is then also convex, lower semicontinuous and coercive. Therefore the infimum of (6.22) is attained; namely, there exists a pair $(\tilde{\sigma}, \tilde{\varepsilon}) \in L^{2}(0, T ; W \times Z)$ such that

$$
A(\sigma, \varepsilon)=\Lambda(\bar{\sigma}, \bar{\varepsilon})=\int_{0}^{T}(T-t) \bar{\sigma}: \bar{\varepsilon}_{t} \mathrm{~d} t
$$

By the definition of $A$ (see (6.21)) and by lemma 6.2 below, this equality also reads

$$
\iint_{\mathcal{Y}_{T}}(T-t)\left\{\varphi(\sigma, y)+\varphi^{*}\left([\varepsilon-B(y): \sigma]_{t}, y\right)-\sigma:[\varepsilon-B(y): \sigma]_{t}\right\} \mathrm{d} y \mathrm{~d} t=0 .
$$

By (2.12)-(2.14) this holds if only if the integrand vanishes a.e. in $\mathcal{Y}_{T}$, and this is equivalent to $(6.25)$.

Finally, note that the minimizing pair $(\tilde{\sigma}, \tilde{\varepsilon})$ is unique whenever $\varphi$ and $\varphi^{*}$ are both strictly convex. We have thus proved theorem 6.3 (stated below, after the lemma). 
Lemma 6.2. If $\sigma^{0} \in W+\mathbb{R}_{\mathrm{s}}^{9}, \varepsilon^{0} \in Z+\mathbb{R}_{\mathrm{s}}^{9}$ and $(\sigma, \varepsilon) \in \Xi\left(\sigma^{0}, \varepsilon^{0}\right)$, then

$$
\begin{aligned}
\iint_{\mathcal{Y}_{T}}(T-t) \sigma:[\varepsilon-B(y): \sigma]_{t} \mathrm{~d} y \mathrm{~d} t \\
=\int_{0}^{T}(T-t) \hat{\sigma}: \hat{\varepsilon}_{t} \mathrm{~d} t \\
\quad-\frac{1}{2} \iint_{\mathcal{Y}_{T}}\left[\sigma(y, t): B(y): \sigma(y, t)-\sigma^{0}(y): B(y): \sigma^{0}(y)\right] \mathrm{d} y \mathrm{~d} t .
\end{aligned}
$$

Proof. Via a standard regularization procedure, one may easily construct three sequences, $\left\{\sigma_{n}^{0} \in W+\mathbb{R}_{\mathrm{s}}^{9}\right\},\left\{\varepsilon_{n}^{0} \in Z+\mathbb{R}_{\mathrm{s}}^{9}\right\}$ and $\left\{\left(\sigma_{n}, \varepsilon_{n}\right) \in L^{2}\left(\mathcal{Y} ; H^{1}(0, T)^{2}\right)\right\}$, such that

$$
\begin{gathered}
\sigma_{n}(0)=\sigma_{n}^{0} \rightarrow \sigma^{0}, \quad \varepsilon_{n}(0)=\varepsilon_{n}^{0} \rightarrow \varepsilon^{0} \quad \text { in } L^{2}(\mathcal{Y})^{9}, \\
\sigma_{n} \rightarrow \sigma, \quad \sqrt{T-t}\left[\varepsilon_{n}-B(y): \sigma_{n}\right]_{t} \rightarrow \sqrt{T-t}[\varepsilon-B(y): \sigma]_{t} \quad \text { in } L^{2}\left(\mathcal{Y}_{T}\right)^{9}, \\
\sqrt{T-t} \hat{\varepsilon}_{n t} \rightarrow \sqrt{T-t} \hat{\varepsilon}_{t} \quad \text { in } L^{2}(0, T)^{9} .
\end{gathered}
$$

Obviously,

$$
\begin{aligned}
& \iint_{\mathcal{Y}_{\tau}} \sigma_{n}:\left[\varepsilon_{n}-B(y): \sigma_{n}\right]_{t} \mathrm{~d} y \mathrm{~d} t \\
& =\iint_{\mathcal{Y}_{\tau}} \sigma_{n}: \varepsilon_{n t} \mathrm{~d} y \mathrm{~d} t-\frac{1}{2} \int_{\mathcal{Y}}\left[\sigma_{n}(y, \tau): B(y): \sigma_{n}(y, \tau)-\sigma^{0}(y): B(y): \sigma^{0}(y)\right] \mathrm{d} y \\
& \quad \text { for all } \tau \in] 0, T] \text { for all } n .
\end{aligned}
$$

As, by $(6.9)_{1}$,

$$
\iint_{\mathcal{Y}_{\tau}} \sigma_{n}: \varepsilon_{n t} \mathrm{~d} y \mathrm{~d} t=\int_{0}^{\tau} \hat{\sigma}_{n}: \hat{\varepsilon}_{n t} \mathrm{~d} t
$$

by integrating once more in time in $] 0, T$ [ we obtain

$$
\begin{aligned}
\iint_{\mathcal{Y}_{T}} & (T-t) \sigma_{n}:\left[\varepsilon_{n}-B(y): \sigma_{n}\right]_{t} \mathrm{~d} y \mathrm{~d} t \\
= & \int_{0}^{T}(T-t) \hat{\sigma}_{n}: \hat{\varepsilon}_{n t} \mathrm{~d} t \\
& \quad-\frac{1}{2} \iint_{\mathcal{Y}_{T}}\left[\sigma_{n}(y, t): B(y): \sigma_{n}(y, t)-\sigma^{0}(y): B(y): \sigma^{0}(y)\right] \mathrm{d} y \mathrm{~d} t \text { for all } n .
\end{aligned}
$$

By passing to the limit in $n$ we then get (6.29).

THEOREM 6.3. Let $p=q=2$, assume that (5.2) and (5.3) are satisfied and define $\Lambda$ as in (6.22). Let also $\sigma^{0} \in W+\mathbb{R}_{\mathrm{s}}^{9}$ and $\varepsilon^{0} \in Z+\mathbb{R}_{\mathrm{s}}^{9}$. Then the following hold.

(i) We have

$$
\Lambda(\bar{\sigma}, \bar{\varepsilon}) \geqslant \int_{0}^{T}(T-t) \bar{\sigma}: \bar{\varepsilon}_{t} \mathrm{~d} t \quad \text { for all }(\bar{\sigma}, \bar{\varepsilon}) \in P\left(\varepsilon^{0}\right) .
$$


(ii) For any $(\sigma, \varepsilon) \in \Xi\left(\sigma^{0}, \varepsilon^{0}\right)$,

$$
[\varepsilon-B(y): \sigma]_{t} \in \partial \varphi(\sigma, y) \quad \text { a.e. in } \mathcal{Y}_{T} \quad \Longrightarrow \quad \Lambda(\hat{\sigma}, \hat{\varepsilon})=\int_{0}^{T}(T-t) \hat{\sigma}: \hat{\varepsilon}_{t} \mathrm{~d} t
$$

(iii) Conversely, for any pair $(\bar{\sigma}, \bar{\varepsilon}) \in P\left(\varepsilon^{0}\right)$, if

$$
\Lambda(\bar{\sigma}, \bar{\varepsilon}) \leqslant \int_{0}^{T}(T-t) \bar{\sigma}: \bar{\varepsilon}_{t} \mathrm{~d} t
$$

then there exists $(\tilde{\sigma}, \tilde{\varepsilon})$ such that $(\sigma, \varepsilon)=(\bar{\sigma}+\tilde{\sigma}, \bar{\varepsilon}+\tilde{\varepsilon}) \in \Sigma\left(\sigma^{0}, \varepsilon^{0}\right)$ and

$$
[\varepsilon-B(y): \sigma]_{t} \in \partial \varphi(\sigma, y) \quad \text { a.e. in } \mathcal{Y}_{T} \text {. }
$$

(iv) If both $\varphi$ and $\varphi^{*}$ are strictly convex, then $(\bar{\sigma}, \bar{\varepsilon})$ uniquely determines $(\tilde{\sigma}, \tilde{\varepsilon})$.

Proposition 6.4 (Euler-Lagrange equations). Let $\sigma^{0} \in W+\mathbb{R}_{\mathrm{s}}^{9}, \varepsilon^{0} \in Z+\mathbb{R}_{\mathrm{s}}^{9}$ and $(\sigma, \varepsilon) \in \Sigma\left(\sigma^{0}, \varepsilon^{0}\right)$. Then $\Lambda(\hat{\sigma}, \hat{\varepsilon})=A(\sigma, \varepsilon)<+\infty$ if and only if (denoting by $B^{*}$ the transpose of the tensor $B)$

$$
\begin{aligned}
& (T-t) \partial \varphi(\sigma(y, t), y)+B(y)^{*}: \\
& \frac{\partial}{\partial t}\left[(T-t) \partial \varphi^{*}\left([\varepsilon-B(y): \sigma]_{t}, y\right)\right]+B(y): \sigma \ni 0 \quad \text { in } \mathcal{D}^{\prime}\left(0, T ; W^{\prime}\right) \\
& \frac{\partial}{\partial t}\left[(T-t) \partial \varphi^{*}\left([\varepsilon-B(y): \sigma]_{t}, y\right)\right] \ni 0 \quad \text { in } \mathcal{D}^{\prime}\left(0, T ; Z^{\prime}\right)
\end{aligned}
$$

\section{REMARK 6.5.}

(i) The functional $\Lambda$ may be compared with the homogenized functional of the calculus of variations (see, for example, $[20,21,31,66]$ ). However, the latter concerns the stationary setting, whereas $\Lambda$ applies to processes.

(ii) By theorem 6.3 the pointwise relation (6.25) is equivalent to the global condition

$$
\begin{aligned}
\Lambda(\hat{\sigma}, \hat{\varepsilon})= & \int_{0}^{T}(T-t) \hat{\sigma}: \hat{\varepsilon}_{t} \mathrm{~d} t \\
& \left(\text { or, equivalently, by }(6.30), \Lambda(\hat{\sigma}, \hat{\varepsilon}) \leqslant \int_{0}^{T}(T-t) \hat{\sigma}: \hat{\varepsilon}_{t} \mathrm{~d} t\right) .
\end{aligned}
$$

It is not clear whether this global-in-time relation may be reformulated as a gradient flow, like (6.33), or otherwise as a pointwise relation in space-time. In this respect, note that after $[95,96]$ memory phenomena are known to arise in the homogenization of linear evolution equations.

(iii) One may show that (6.35) represents a monotone relation between $\hat{\sigma}$ and $\hat{\varepsilon}_{t}$. This issue will be studied separately. 
(iv) If $B$ and $\varphi$ do not depend on $y$, then by Jensen's inequality for any $(\bar{\sigma}, \bar{\varepsilon}) \in$ $P\left(\varepsilon^{0}\right)$ the infimum of $(6.22)$ is attained for $\tilde{\sigma}=\tilde{\varepsilon}=0$ identically in $\mathcal{Y}_{T}$, whence $\Lambda(\bar{\sigma}, \bar{\varepsilon})=A(\sigma, \varepsilon)$. The inequality (6.35) is then equivalent to the relation $(\bar{\varepsilon}-B: \bar{\sigma})_{t} \in \partial \varphi(\bar{\sigma})$ a.e. in $] 0, T[$.

\section{Single-scale homogenization}

In this section we complete the homogenization procedure by eliminating any dependence on the fine-scale variable $y \in \mathcal{Y}$ in the two-scale problem 5.1, and thus derive a purely coarse-scale formulation (upscaling). Conversely, we also retrieve problem 5.1 from this single-scale problem.

We still assume that $p=q=2$ and that (5.1)-(5.3) are satisfied, so that we can define $\Lambda$ as in (6.22). We also assume that

$$
\begin{array}{r}
\boldsymbol{f} \in L^{2}\left(\Omega_{T}\right)^{3}, \quad \boldsymbol{u}^{0} \in L^{2}(\Omega)^{3} \cap V, \quad \boldsymbol{v}^{0} \in L^{2}(\Omega)^{3}, \quad \bar{\sigma}^{0} \in L^{2}(\Omega)_{\mathrm{s}}^{9} \\
\text { such that, setting } \bar{\varepsilon}^{0}:=\nabla^{\mathrm{s}} \boldsymbol{u}^{0}, \quad \bar{\varepsilon}_{(\mathrm{s})}^{0}=\beta \sigma_{(\mathrm{s})}^{0} .
\end{array}
$$

We can now introduce a single-scale problem in weak form.

Problem 7.1. Find $(\boldsymbol{u}, \bar{\sigma})$ such that, setting $\bar{\varepsilon}:=\nabla^{\mathrm{s}} \boldsymbol{u}$ and defining $\Lambda$ as in (6.22) with $\bar{\sigma}^{0}$ in place of $\sigma^{0}$, we obtain

$$
\begin{gathered}
\boldsymbol{u} \in H^{1}\left(0, T ; L^{2}(\Omega)^{3}\right) \cap L^{2}(0, T ; V), \quad \bar{\sigma} \in L^{2}\left(0, T ; L^{2}(\Omega)_{\mathrm{s}}^{9}\right) \\
\frac{1}{2} \iint_{\Omega_{T}} \rho\left(\left|\frac{\partial \boldsymbol{u}}{\partial t}(x, t)\right|^{2}-\left|\boldsymbol{v}^{0}(x)\right|^{2}\right) \mathrm{d} x \mathrm{~d} t+\int_{\Omega} \Lambda(\bar{\sigma}, \bar{\varepsilon}) \mathrm{d} x \\
\quad \leqslant \iint_{\Omega_{T}}(T-t) \boldsymbol{f} \cdot \frac{\partial \boldsymbol{u}}{\partial t} \mathrm{~d} x \mathrm{~d} t \\
\iint_{\Omega_{T}}\left\{\rho\left(\boldsymbol{u}^{0}-\boldsymbol{u}\right) \cdot \frac{\partial \boldsymbol{w}}{\partial t}+\int_{0}^{t} \bar{\sigma}(\cdot, \tau) \mathrm{d} \tau: \nabla \boldsymbol{w}\right\} \mathrm{d} x \mathrm{~d} t \\
=\iint_{\Omega_{T}}\left(\int_{0}^{t} \boldsymbol{f}(\cdot, \tau) \mathrm{d} \tau+\rho \boldsymbol{v}^{0}\right) \cdot \boldsymbol{w} \mathrm{d} x \mathrm{~d} t \\
\quad \text { for all } \boldsymbol{w} \in H^{1}(0, T ; V), \boldsymbol{w}(\cdot, T)=\mathbf{0} \text { a.e. in } \Omega
\end{gathered}
$$

Note that the initial condition on $\bar{\varepsilon}$ is implicit in the definition of the functional $\Lambda$ (see (6.21) and (6.22)).

Theorem 7.2 (relation between the two-scale and single-scale problems). Let $p=$ $q=2$ and assume that (5.1)-(5.3) and (7.1) are satisfied. Define $\Lambda$ as in (6.22). Then

(i) if $(\boldsymbol{u}, \varepsilon, \sigma)$ is a solution of problem 5.1, then $(\boldsymbol{u}, \hat{\sigma})$ solves problem 7.1;

(ii) conversely, for any solution $(\boldsymbol{u}, \bar{\sigma})$ of problem 7.1 such that

$$
\boldsymbol{u} \in H^{2}\left(0, T ; L^{2}(\Omega)^{3}\right) \cap H^{1}(0, T ; V), \quad \sigma \in W^{1,1}\left(0, T ; L^{2}(\Omega)^{3}\right),
$$

there exist $\varepsilon, \sigma$ such that $(\boldsymbol{u}, \varepsilon, \sigma)$ solves problem 5.1 and $\hat{\varepsilon}=\bar{\varepsilon}, \hat{\sigma}=\bar{\sigma}$. 
The latter statement is not empty, for by theorem 5.6 and by theorem $7.2(\mathrm{i})$ there exists a solution of problem 7.1 with the regularity (7.5), under suitable hypotheses on the data.

Although upscaling from a two-scale to a single-scale problem may be expected to entail some loss of information, by theorem 7.2(ii) no spurious solution is introduced in this way.

Proof.

(i) By the definition (6.22) of $\Lambda$,

$$
\begin{aligned}
& \int_{\Omega} \Lambda(\hat{\sigma}, \hat{\varepsilon}) \mathrm{d} x \\
& \leqslant \iiint_{\Omega_{T} \times \mathcal{Y}}\left\{( T - t ) \left[\varphi(\sigma(x, y, t), y)+\varphi^{*}\left(\frac{\partial}{\partial t}[\varepsilon-B((y): \sigma](x, y, t), y)\right]\right.\right. \\
& +\frac{1}{2} \sigma(x, y, t): B((y): \sigma(x, y, t) \\
& \quad-\frac{1}{2} \sigma^{0}(x, y): B\left((y): \sigma^{0}(x, y)\right\} \mathrm{d} x \mathrm{~d} y \mathrm{~d} t .
\end{aligned}
$$

Integrating (5.10) in time in $] 0, T[$ and then inserting this inequality we get (7.3). The pair $(\boldsymbol{u}, \hat{\sigma})$ thus solves problem 7.1 .

(ii) By the discussion of $\S 3$ we know that, under the further regularity (7.5), the equation of continuum dynamics (3.20) (here with $\bar{\sigma}$ and $\boldsymbol{f}$ in place of $\sigma$ and $\boldsymbol{f}_{1}$ ) is satisfied a.e. in $\Omega_{T}$. Multiplying this equation by $\partial \boldsymbol{u} / \partial t$ and integrating twice in time in $] 0, T[$, we then get

$\iint_{\Omega_{T}}\left(\frac{\rho}{2}\left|\frac{\partial \boldsymbol{u}}{\partial t}(x, t)\right|^{2}-\frac{\rho}{2}\left|\boldsymbol{v}^{0}(x)\right|^{2}+(T-t) \bar{\sigma}: \frac{\partial \bar{\varepsilon}}{\partial t}\right) \mathrm{d} x \mathrm{~d} t=\iint_{\Omega_{T}}(T-t) \boldsymbol{f} \cdot \frac{\partial \boldsymbol{u}}{\partial t} \mathrm{~d} x \mathrm{~d} t$

so that by comparing this equality with (7.3) we infer that

$$
\left.\left.\int_{\Omega} \Lambda(\bar{\sigma}, \bar{\varepsilon}) \mathrm{d} x \leqslant \iint_{\Omega_{T}}(T-t) \bar{\sigma}: \frac{\partial \bar{\varepsilon}}{\partial t} \mathrm{~d} x \mathrm{~d} t \quad \text { for a.e. } t \in\right] 0, T\right] .
$$

By (6.30) the inequality (6.32) then holds for a.e. $x \in \Omega$. By (7.2) and theorem 6.3 (iii) there then exist $(\tilde{\sigma}, \tilde{\varepsilon})$ such that $(\sigma, \varepsilon)=(\bar{\sigma}+\tilde{\sigma}, \bar{\varepsilon}+\tilde{\varepsilon})$ satisfies $(6.33)$ a.e. in $\Omega \times \mathcal{Y}_{T}$. In $\S 5$ we saw that this yields (5.10). The triplet $(\boldsymbol{u}, \varepsilon, \sigma)$ thus solves problem 5.1 and $\hat{\varepsilon}=\bar{\varepsilon}, \hat{\sigma}=\bar{\sigma}$.

By the next statement problem 7.1 may be regarded as the homogenized formulation of problem $3.1_{\eta}$.

COROllary 7.3. Let the hypotheses of theorem 7.2 hold, and for any $\eta>0$ let $\left(\boldsymbol{u}_{\eta}, \sigma_{\eta}\right)$ be a solution of problem $3.1_{\eta}$ that satisfies the estimates (5.16). Then there exist $\boldsymbol{u}$ and $\bar{\sigma}$ such that, as $\eta \rightarrow 0$ along a suitable sequence,

$$
\begin{aligned}
& \boldsymbol{u}_{\eta} \stackrel{*}{\rightarrow} \boldsymbol{u} \quad \text { in } W^{1, \infty}\left(0, T ; L^{2}(\Omega)^{3}\right) \cap L^{2}(0, T ; V), \\
& \sigma_{\eta} \stackrel{*}{\rightarrow} \bar{\sigma} \quad \text { in } L^{\infty}\left(0, T ; L^{2}(\Omega)^{9}\right) .
\end{aligned}
$$

This implies that $(\boldsymbol{u}, \bar{\sigma})$ is a solution of problem 7.1 . 
Proof. Let us recall theorem 5.5, and note that (5.22) and (5.24) yield (7.7) and (7.8). The final statement follows from theorem 7.2(i).

REMARK 7.4.

(i) The limit behaviour of problem 7.1 as $\rho \rightarrow 0$ mimics that of $\S 5$.

(ii) By theorem 6.3 and by remark 6.5(iv), if $B$ and $\varphi$ do not depend on $y$, then (7.3) is equivalent to the inclusion (3.5). In this case problem 3.1 is thus equivalent to problem 7.1.

(iii) The above multi-scale homogenization procedure may be adapted to mixtures of elastic and viscous materials, as we outlined in the limit case (v) at the end of $\S 4$.

\section{Conclusions and further questions}

\subsection{Is the generalized Maxwell model justifiable?}

At the basis of the nonlinear model (1.3) of viscoelasticity of fluid type there is the mean-field-type hypothesis that the strain should be uniform at a fine length scale. As we have seen, this also encompasses the Prandtl-Reuss model of elastoplasticity as a limit case. Our analysis does not show any reason to expect such a uniformity condition to hold, and clearly one cannot append a further constraint like this to the model. Analogous conclusions are known to apply to stationary models of continuum mechanics.

The fine-scale non-uniformity of the strain is essentially due to the lack of a uniform $W^{s, p}(\Omega)$-type estimate for any $s>0$ for the approximating family $\left\{\varepsilon_{\eta}: \eta>\right.$ $0\}$. The occurrence of high gradients for these fields and the onset of a non-trivial mesoscopic structure can thus hardly be ruled out. In the Hilbertian framework, namely, for $p=q=2$, a single-scale homogenized model was nevertheless retrieved via two basic ingredients: the mutual orthogonality of the spaces $W$ and $Z$ (see $(6.7)-(6.9))$ and the convexity of the potential $\varphi$, which we exploited via the Fenchel properties (2.12) and (2.13).

\subsection{Two-scale and single-scale homogenization}

In this work we proceeded along the following lines, which can also be applied to the homogenization of other either stationary or evolutive nonlinear phenomena (see $[102,103,106,107])$.

(i) First we constructed a single-scale model, $P$, for an inhomogeneous material, assuming that the fields only depend on the coarse-scale variable $x$ (besides time), and proved the existence and uniqueness of the weak solution.

(ii) We represented a composite by assuming that the constitutive data $B$ and $\varphi$ depend periodically on a fine-scale variable $y:=x / \eta$, and formulated a corresponding single-scale initial- and boundary-value problem, $P_{\eta}$. Existence and uniqueness of the weak solution followed from the previous step. 
(iii) We showed that as $\eta \rightarrow 0$ a subsequence of solutions of $P_{\eta}$ weakly two-scale converges to a solution of a two-scale problem, $P_{2}$, in which the fields depend on both the coarse-scale and fine-scale variables $x, y$.

(iv) For $p=q=2$ we derived a coarse-scale relation from the two-scale constitutive law. We also showed that, conversely, all fields that satisfy that coarsescale relation may be retrieved as averages of fields that satisfy the two-scale constitutive law.

(v) We extended these scale-transformations to our initial- and boundary-value problem and derived a single-scale problem $P_{1}$ from the two-scale problem $P_{2}$, essentially by averaging the mesoscopic fields over the reference set $\mathcal{Y}$.

(vi) We showed that conversely any solution of $P_{1}$ can be represented as the $\mathcal{Y}$ average of a solution of problem $P_{2}$. Thus, no spurious solution may be introduced by the upscaling procedure.

\subsection{Further questions}

(i) In $\S \S 6$ and 7 we assumed that $p=q=2$, for we do not know whether $\sigma \in L^{p}\left(\Omega_{T} \times \mathcal{Y}\right)_{\mathrm{s}}^{9}$ for $p>2$. The extension of the upscaling procedure to $q<2<p$ would of course be interesting; the case of $q=1$ and $p=\infty$ would be especially relevant, as it encompasses elasto-viscoplasticity and, in particular, the Prandtl-Reuss model.

(ii) The weak formulation of $\S 3$ and its analysis can be extended to the case in which the prescribed functions $B(x)$ and $\varphi(\cdot, x)$ also depend on time. It may be of some interest to apply the procedure of two-scale convergence to the corresponding time homogenization. In view of applications, it may also be useful to allow for purely viscous and/or purely elastic parts of the body, by allowing $B$ and/or $\varphi$ to degenerate in subdomains of $\Omega$; a similar issue is addressed in [106].

(iii) It is known that De Giorgi's $\Gamma$-convergence provides a unifying framework for the homogenization of stationary problems (see, for example, $[20,21,31]$ ). In [106] this notion is also applied to the homogenization of an evolution problem; after that, this is reformulated as a minimization principle. That approach may be applied to the system (1.1), (1.2).

(iv) In $\S 1$ we outlined the Kelvin-Voigt and Maxwell models. A more general model of elasto-viscoplasticity is represented by an inclusion of the form

$$
\frac{\mathrm{d} \varepsilon}{\mathrm{d} t}-B: \frac{\mathrm{d} \sigma}{\mathrm{d} t} \in \partial \varphi(\sigma-A: \varepsilon) \quad \text { (with } A, B \in \mathbb{R}^{3^{4}} \text { positive semi-definite). }
$$

For $A=0(B=0$, respectively) we retrieve the Maxwell (Kelvin-Voigt, respectively) model. If $\varphi=I_{K}^{*}$ is the support function of a closed convex $K \subset D_{\mathrm{s}}^{9}$ that contains the origin, (8.1) accounts for elastoplasticity with kinematic strain-hardening. This constitutive relation was coupled with the equation of continuum dynamics in $[13,14,61]$, where well-posedness was proved. The homogenization procedure that we have illustrated in the present paper may also be applied to this problem. 


\section{Acknowledgments}

This research was partly supported by the PRIN projects 'Mathematical modelling and analysis of free boundary problems' (2004-2005) and 'Free boundary problems, phase transitions and models of hysteresis' (2006-2007) of the Italian Ministero dell'Università e della Ricerca.

The author gratefully acknowledges several useful remarks of the anonymous reviewer.

\section{References}

1 H.-D. Alber. Global existence and boundedness of large solutions to nonlinear equations of viscoelasticity with hardening. Commun. Math. Phys. 166 (1995), 565-601.

2 H.-D. Alber. Materials with memory (Springer, 1998).

3 H.-D. Alber. Justification of homogenized models for viscoplastic bodies with microstructure. In Deformation and failure in metallic materials (ed. K. Hutter and H. Baaser), pp. 295-319 (Springer, 2003).

4 G. Allaire. Homogenization and two-scale convergence. SIAM J. Math. Analysis 23 (1992), 1482-1518.

5 G. Allaire. Shape optimization by the homogenization method (Springer, 2002).

6 M. Amar. Two-scale convergence and homogenization on $\operatorname{BV}(\Omega)$. Asymp. Analysis 16 (1998), 65-84.

7 L. Ambrosio, A. Coscia and G. Dal Maso. Fine properties of functions with bounded deformation. Arch. Ration. Mech. Analysis 139 (1997), 201-238.

8 L. Ambrosio, N. Fusco and D. Pallara. Functions of bounded variation and free discontinuity problems (Clarendon Press, 2000).

9 S. S. Antman. Nonlinear problems of elasticity (Springer, 2005).

10 G. Anzellotti. On the existence of the rates of stress and displacement for Prandtl-Reuss plasticity. Q. Appl. Math. 41 (1983), 181-208.

11 G. Anzellotti and S. Luckhaus. Dynamical evolution of elasto-perfectly plastic bodies. Appl. Math. Optim. 15 (1987), 121-140.

12 G. Bensoussan, J. L. Lions and G. Papanicolaou. Asymptotic analysis for periodic structures (Amsterdam: North-Holland, 1978).

13 D. Blanchard and P. Le Tallec. Numerical analysis of the equations of small strains quasistatic elastoviscoplasticity. Numer. Math. 50 (1986), 147-169.

14 D. Blanchard, P. Le Tallec and M. Ravachol. Numerical analysis of evolution problems in nonlinear small strains elastoviscoplasticity. Numer. Math. 55 (1989), 177-195.

15 G. Bouchitté and G. Dal Maso. Integral representation and relaxation of convex local functionals on BV $(\Omega)$. Annali Scuola Norm. Sup. Pisa IV 20 (1993), 483-533.

16 G. Bouchitté and P. Suquet. Charges limites, plasticité et homogénéisation: le cas d'un bord chargé. C. R. Acad. Sci. Paris Sér. I 305 (1987), 441-444.

17 G. Bouchitté and P. Suquet. Homogenization, plasticity and yield design. In Composite media and homogenization theory (ed. G. dal Maso and G. F. Dell'Antonio), pp. 107-133. (Birkhäuser, 1991).

18 G. Bouchittté. Convergence et relaxation de fonctionnelles du calcul des variations á croissance linéaire. Application à l'homogénéisation en plasticité. Annales Fac. Sci. Toulouse Math. 8 (1986), 7-36.

19 G. Bouchittté. Représentation intégrale de fonctionnelles convexes sur un espace de mesures. II. Cas de l'épi-convergence. Ann. Univ. Ferrara Sci. Mat. 33 (1987), 113-156.

20 A. Braides. $\Gamma$-convergence for beginners (Oxford University Press, 2002).

21 A. Braides and A. Defranceschi. Homogenization of multiple integrals (Oxford University Press, 1998).

22 H. Brezis and I. Ekeland. Un principe variationnel associé à certaines équations paraboliques. I. Le cas indépendant du temps. C. R. Acad. Sci. Paris Sér. A-B 282 (1976), 971-974. 
H. Brezis and I. Ekeland. Un principe variationnel associé à certaines équations paraboliques. II. Le cas dépendant du temps. C. R. Acad. Sci. Paris Sér. A-B 282 (1976), 1197-1198.

M. Brokate and J. Sprekels. Hysteresis and phase transitions (Springer, 1996).

G. Buttazzo. Semicontinuity, relaxation and integral representation in the calculus of variations (Harlow: Longman, 1989).

26 C. Carstensen. Interface problems in viscoplasticity and plasticity. SIAM J. Math. Analysis 25 (1994), 1468-1487.

A. Cherkaev and R. Kohn (eds). Topics in the mathematical modelling of composite materials (Birkhäuser, 1997).

V. Chiadò Piat and G. V. Sandrakov. Homogenization of some variational inequalities for elastoplastic torsion problems. Asymp. Analysis 40 (2004), 1-23.

E. Christiansen. On the collapse solution in limit analysis. Arch. Ration. Mech. Analysis 91 (1985), 119-135.

. Cioranescu and P. Donato. An introduction to homogenization (Oxford University Press, 1999).

31 G. Dal Maso. An introduction to $\Gamma$-convergence (Birkhäuser, 1993).

32 G. Dal Maso, A. De Simone and M. G. Mora. Quasistatic evolution problems for linearly elastic-perfectly plastic materials. Arch. Ration. Mech. Analysis 180 (2006), 237-291.

33 E. De Giorgi and T. Franzoni. Su un tipo di convergenza variazionale. Atti Accad. Naz. Lincei Rend. Cl. Sci. Fis. Mat. Natur. 58 (1975), 842-850.

34 E. De Giorgi and S. Spagnolo. Sulla convergenza degli integrali dell'energia per operatori ellittici del secondo ordine. Boll. UMI 8 (1973), 391-411.

35 J. J. F. Demengel and T. Qi. Convex function of a measure obtained by homogenization. SIAM J. Math. Analysis 21 (1990), 409-435.

36 G. Duvaut and J. L. Lions. Les inéquations en mécanique et en physique (Paris: Dunod, 1972).

E. Homogenization of linear and nonlinear transport equations. Commun. Pure Appl. Math. 45 (1992), 301-326.

38 S. Ebenfeld. Remarks on the quasistatic problem of viscoelasticity: existence, uniqueness and homogenization. Continuum Mech. Thermodyn. 14 (2002), 511-526.

39 I. Ekeland and R. Temam. Analyse convexe et problèmes variationnelles (Paris: Dunod Gauthier-Villars, 1974).

40 R. A. Eve, B. D. Reddy and R. T. Rockafellar. An internal variable theory of elastoplasticity based on the maximum plastic work inequality. Q. Appl. Math. 48 (1990), 59-83.

41 W. Fenchel. Convex cones, sets, and functions (Princeton University Press, 1953).

42 W. Flügge. Viscoelasticity (Springer, 1975).

43 J. Franců and P. Krejčí. Homogenization of scalar wave equations with hysteresis. Continuum Mech. Thermodyn. 11 (1999), 371-390.

44 G. Geymonat and P. Suquet. Functional spaces for Norton-Hoff materials. Math. Meth. Appl. Sci. 8 (1986), 206-222.

45 C. Goffman and J. Serrin. Sublinear functions of measures and variational integrals. Duke Math. J. 31 (1964), 159-178.

46 M. E. Gurtin. The linear theory of elasticity. In Handbuch der Physik (ed. S. Flügge), vol. VIa/2, pp. 1-295 (Springer, 1972).

47 B. Halphen and N. Q. Son. Sur les matériaux standard généralisés. J. Méc. 14 (1975), 39-63.

48 W. Han and B. D. Reddy. Plasticity (Springer, 1999).

49 P. Haupt. Continuum mechanics and theory of materials (Springer, 2001).

50 J.-B. Hiriart-Urruty and C. Lemarechal. Convex analysis and optimization algorithms (Springer, 1993).

51 U. Hornung (ed.). Homogenization and porous media (Springer, 1997).

52 A. D. Ioffe and V. M. Tihomirov. Theory of extremal problems (Amsterdam: North-Holland, 1979).

53 V. V. Jikov, S. M. Kozlov and O. A. Oleinik. Homogenization of differential operators and integral functionals (Springer, 1994). 
54 C. Johnson. Existence theorems for plasticity problems. J. Math. Pures Appl. 55 (1976), 431-444. Cohnson. On plasticity with hardening. J. Math. Analysis Applic. 62 (1978), 325-336. A. M. Khludnev and J. Sokolowski. On solvability of boundary value problems in elastoplasticity. Control Cybernet. 27 (1998), 311-330.

57 R. V. Kohn and T. D. Little. Some model problems of polycrystal plasticity with deficient basic crystals. SIAM J. Appl. Math. 59 (1999), 172-197.

58 W. T. Koiter. General theorems for elastic-plastic solids. In Progress in solid mechanics vol. 6 (ed. I. N. Sneddon and R. Hill), pp. 167-221 (Amsterdam: North-Holland, 1960). M. A. Krasnosel'skiı̌ and A. V. Pokrovskiu. Systems with hysteresis (Springer, 1989). P. Krejčí. Convexity, hysteresis and dissipation in hyperbolic equations (Tokyo: Gakkotosho, 1996).

61 P. Le Tallec. Numerical analysis of viscoelastic problems (Paris: Masson, 1990).

62 M. J. Leitman and G. M. C. Fisher. The linear theory of viscoelasticity. In Handbuch der Physik (ed. S. Flügge), vol. VIa/3, pp. 1-123 (Springer, 1973). J. Lemaitre and J.-L. Chaboche. Mechanics of solid materials (Cambridge University Press, 1990).

64 J. Lubliner. Plasticity theory (New York: Macmillan, 1990).

65 D. Lukkassen, G. Nguetseng and P. Wall. Two-scale convergence. Int. J. Pure Appl. Math. 2 (2002), 35-86.

66 P. Marcellini. Periodic solutions and homogenization of nonlinear variational problems. Annali Mat. Pura Appl. 117 (1978), 139-152.

67 G. A. Maugin. The thermodynamics of plasticity and fracture (Cambridge University Press, 1992).

68 A. Mielke. Energetic formulation of multiplicative elastoplasticity using dissipation distances. Continuum Mech. Thermodyn. 15 (2003), 351-382.

69 A. Mielke. Existence of minimizers in incremental elastoplasticity with finite strains. SIAM J. Math. Analysis 36 (2004), 384-404.

70 A. Mielke and A. Timofte. Two-scale homogenization for evolutionary variational inequalities via the energetic formulation. SIAM J. Math. Analysis 39 (2007), 642-668.

71 G. W. Milton. The theory of composites (Cambridge University Press, 2002).

72 J. J. Moreau. Fonctionnelles convexes, Séminaires sur les équations aux derivées partielles (Paris: Collège de France, 1967).

73 J. J. Moreau. Sur les lois de frottement, de plastictité et de viscosité. C. R. Acad. Sci. Paris Sér. II 271 (1970), 608-611.

74 J. J. Moreau. Application of convex analysis to the treatment of elastoplastic systems. In Applications of methods of functional analysis to problems in mechanics (ed. P. Germain and B. Nayroles), pp. 56-89 (Springer, 1976).

F. Murat. Compacité par compensation. Annali Scuola Norm. Sup. Pisa 5 (1978), 489-507. F. Murat and L. Tartar. H-convergence. In Topics in the mathematical modelling of composite materials (ed. A. Cherkaev and R. Kohn), pp. 21-44 (Birkhäuser, 1997).

77 A. K. Nandakumaran and M. Rajesh. Homogenization of a nonlinear degenerate parabolic differential equation. Electron. J. Diff. Eqns 2001 (2001), 1-19.

78 S. Nesenenko. Homogenization in viscoplasticity. SIAM J. Math. Analysis 39 (2007), 236262.

79 J. Nečas and I. Hlaváček. Mathematical theory of elastic and elasto-plastic bodies: an introduction (Elsevier, 1982).

80 G. Nguetseng. A general convergence result for a functional related to the theory of homogenization. SIAM J. Math. Analysis 20 (1989), 608-623.

81 W. Prager. An introduction to mechanics of continua (Boston, MA: Ginn, 1961).

82 M. Reiner. Rheology. In Handbuch der Physik (ed. S. Flügge), vol. VI, pp. 434-550 (Springer, 1958).

83 M. Renardy, W. J. Hrusa and J. A. Nohel. Mathematical problems in viscoelasticity (Harlow: Longman Scientific and Technical, 1987).

84 R. T. Rockafellar. Convex analysis (Princeton University Press, 1969).

85 E. Sanchez-Palencia. Non-homogeneous media and vibration theory (Springer, 1980).

86 J. C. Simo and T. J. R. Hughes. Computational inelasticity (Springer, 1998). 
S. Spagnolo. Sulla convergenza di soluzioni di equazioni paraboliche ed ellittiche. Annali Scuola Norm. Sup. Pisa III 22 (1968), 571-597. (Errata. Annali Scuola Norm. Sup. Pisa III 22 (1968), 673.)

G. Strang and R. Temam. Functions of bounded deformation. Arch. Ration. Mech. Analysis 75 (1980), 7-21.

89 P. Suquet. Existence et régularité des solutions des équations de la plasticité parfaite. $C$. R. Acad. Sci. Paris Sér. D 286 (1978), 1201-1204.

90 P. Suquet. Evolution problems for a class of dissipative materials. Q. Appl. Math. 38 (1980), 391-414.

91 P. Suquet. Sur les équations de la plasticité: existence et regularité des solutions. J. Méc. 20 (1981), 3-39.

92 P. Suquet. Plasticité et homogéneization. Thèse de doctorat d'état (Paris, 1982).

93 P. Suquet. Analyse limite et homogénéisation. C. R. Acad. Sci. Paris II 296 (1983), 13551358.

94 L. Tartar. Course Peccot, Collège de France, Paris (1977). (Unpublished, partially reproduced in [27, pp. 21-44].)

95 L. Tartar. Nonlocal effects induced by homogenization. In Partial differential equations and the calculus of variations, vol. II (ed. F. Colombini, A. Marino, L. Modica and S. Spagnolo), pp. 925-938 (Birkhäuser, 1989).

96 L. Tartar. Memory effects and homogenization. Arch. Ration. Mech. Analysis 111 (1990), 121-133.

97 R. Temam. Problèmes mathématiques en plasticité (Paris: Gauthier-Villars, 1983).

98 R. Temam. A generalized Norton-Hoff model and the Prandtl-Reuss law of plasticity. Arch. Ration. Mech. Analysis 95 (1986), 137-183.

99 M. Valadier. Admissible functions in two-scale convergence. Portugaliae Math. 54 (1997), 147-164.

100 A. Visintin. Differential models of hysteresis (Springer, 1994).

101 A. Visintin. Some properties of two-scale convergence. Rend. Accad. Lincei 15 (2004), 93-107.

102 A. Visintin. Homogenization of doubly nonlinear equations. Rend. Lincei Mat. Appl. 17 (2006), 211-222.

103 A. Visintin. Homogenization of the nonlinear Kelvin-Voigt model of viscoelasticity and of the Prager model of plasticity. Continuum Mech. Thermodyn. 18 (2006), 223-252.

104 A. Visintin. Two-scale convergence of first-order operators. Z. Analysis Anwend. 26 (2007), 133-164.

105 A. Visintin. Two-scale convergence of some integral functionals. Calc. Var. PDEs 29 (2007), 239-265.

106 A. Visintin. Homogenization of nonlinear visco-elastic composites. J. Math. Pures Appl. 89 (2008), 477-504.

107 A. Visintin. Homogenization of processes in nonlinear hereditary composites. (In preparation.)

108 V. V. Zhikov. On an extension and an application of the two-scale convergence method. Mat. Sb. 191 (2000), 31-72. (In Russian.)

(Issued Publication date 2008) 
